\title{
Analytic Gradients of Approximate Coupled Cluster Methods with Quadruple Excitations
}

\author{
Devin A. Matthews* \\ Southern Methodist University, Dallas, TX 75275, USA \\ E-mail: damatthews@smu.edu
}

\begin{abstract}
The analytic gradient theory for both iterative and non-iterative coupled-cluster approximations that include connected quadruple excitations is presented. These methods include, in particular, CCSDT(Q), which is an analog of the well-known CCSD(T) method which starts from the full CCSDT method rather than CCSD. The resulting methods are implemented in the CFOUR program suite, and pilot applications are presented for the equilibrium geometries and harmonic vibrational frequencies of the simplest Criegee intermediate, $\mathrm{CH}_{2} \mathrm{OO}$, as well as to the isomerization pathway between dimethylcarbene and propene. While all methods are seen to approximate the full CCSDTQ results well for "well-behaved" systems, the more difficult case of the Criegee intermediate shows that $\operatorname{CCSDT}(\mathrm{Q})$, as well as certain iterative approximations, display problematic behavior.
\end{abstract}

\section{Introduction}

The inclusion of higher-order correlation effects has been recognized as an important contribution to the calculation of accurate molecular energies for more than a decade. $\frac{1-\underline{5}}{\underline{5}}$ Such contributions are critical to the accurate evaluation of molecular and reaction enthalpies, $\underline{\underline{6}} \underline{\underline{8}}$ 
atomization energies, $, \underline{9}, \underline{10}$ barrier heights, $\underline{\underline{11}}$ and intermolecular interaction energies,,$\underline{12}$ and are included in many standard thermochemical model chemistries such as HEAT, $\underline{13} \underline{-16}$ Wn, $\underline{\underline{17}-19}$ and ANL- $n . \underline{20}$ However, the effect of higher-order electron correlation on equilibrium molecular geometries, vibrational frequencies, and other properties is less well-understood. Including geometric effects beyond the $\operatorname{CCSD}(\mathrm{T})^{21}$ or $\mathrm{CCSDT}^{22}$ level could be desirable in a number of circumstances. For example, Morgan et al. calculated the equilibrium geometry of formaldehyde including $\operatorname{CCSDT}(\mathrm{Q})^{23}$ and $\mathrm{CCSDTQ}^{24} \underline{-26}$ higher-order correlation contributions, as well as anharmonic vibrational frequencies including CCSDT(Q) corrections. ${ }^{27}$ Puzzarini et al. investigated the effect of higher-order correlation on computed rotational constants. $\stackrel{28}{ }$ Heckert et al. computed the higher-order correlation contribution to the geometry of several small molecules, and found rather large changes in geometry especially for triply bonded species such as $\mathrm{N}_{2}, \mathrm{HCN}$, and $\mathrm{HCCH}$, as well as for $\mathrm{F}_{2} \cdot \underline{29}, \underline{30}$ Ruden et al. investigated the contribution of quadruple excitations to the harmonic frequencies of several diatomics and found corrections on going from CCSDT to CCSDTQ as large as $20 \mathrm{~cm}^{-1} . \underline{31}$

The high computational expense of CCSDTQ naturally limits applicability to small molecules. Approximate coupled cluster methods would ideally extend the range of applicability, especially for non-iterative approximations such as CCSDT(Q). However, for geometric derivatives and especially for harmonic frequencies, the lack of analytic gradients further increases the cost as finite-difference methods must be used. This additional expense furthermore scales with the molecular size rather prohibitively as the finite difference technique depends on the number of degrees of freedom while analytic gradients do not. $\underline{32}$ In order to efficiently study the effect of such higher-order effects on molecular properties, we present here the derivation of analytic gradients for a number of approximate coupled cluster methods that include connected quadruple excitations: $\operatorname{CCSDT}(\mathrm{Q})^{\underline{23}}$ (and its A and B variants $\left.{ }^{33}\right)$, CCSDTQ-1a, $-1 \mathrm{~b}$, and $-3, \underline{34}$ and CC4. $\underline{34}$ While analytic gradients for general coupled cluster models, including CCSDT $\stackrel{35}{5}$ and CCSDTQ, $\underline{\underline{36}}$ have been available for some time, analytic gradients for general approximate coupled cluster methods $\frac{34}{}$ have not 
as yet been derived, except for the special cases of CCSDT- $n, \underline{37,38}$ CC $3, \underline{38}$ and of course $\operatorname{CCSD}(\mathrm{T}) \cdot \underline{39}, \underline{40}$

\section{Theory}

The theory of analytic CCSDT(Q) gradients is developed by first reviewing the basic theory of coupled cluster and its gradients. $\underline{32,41}$ Then, the derivation of the CCSDT(Q) energy is reviewed and considerations for non-Hartree Fock references (such as ROHF and QRHF) are discussed. These theories are then combined to derive explicit CCSDT(Q) gradient expressions. Next, iterative approximations to the CCSDTQ energy are reviewed, and finally the corresponding analytic gradient theories are developed.

\subsection{Coupled Cluster Gradients}

The coupled cluster energy is conveniently written as a matrix element of the coupled cluster transformed Hamiltonian, $\bar{H}=e^{-\hat{T}} \hat{H} e^{\hat{T}}=\left(\hat{H} e^{\hat{T}}\right)_{c}^{\underline{\underline{44}} \underline{\underline{46}}}$

$$
E_{C C}=\langle 0|\bar{H}| 0\rangle
$$

where $\hat{H}$ is the Hamiltonian in the molecular orbital basis, normal-ordered with respect to the reference single-particle wavefunction $|0\rangle$, and the cluster operator $\hat{T}$ is an excitation operator,

$$
\begin{aligned}
& \hat{H}=\hat{F}+\hat{V}=\sum_{p q} f_{q}^{p}\left\{p^{\dagger} q\right\}+\frac{1}{4} \sum_{p q r s} v_{r s}^{p q}\left\{p^{\dagger} q^{\dagger} s r\right\} \\
& \hat{T}=\sum_{k=1}^{N} \hat{T}_{k}=\sum_{k=1}^{N} \frac{1}{(k !)^{2}} \sum_{\substack{a_{1} \ldots a_{k} \\
i_{1} \ldots i_{k}}} t_{i_{1} \ldots i_{k}}^{a_{1} \ldots a_{k}} a_{1}^{\dagger} \ldots a_{k}^{\dagger} i_{k} \ldots i_{1}
\end{aligned}
$$

for occupied spin-orbitals $i j \ldots$, virtual (unoccupied) spin-orbitals $a b \ldots$, and arbitrary spinorbitals pqrs, and where $\{\ldots\}$ denotes normal ordering. The number of excitations $N$ 
included in the cluster operator gives a hierarchy of coupled cluster methods, CCSD $(N=2)$ $\rightarrow$ CCSDT $\rightarrow$ CCSDTQ $\rightarrow \ldots \rightarrow$ Full Coupled Cluster (FCC), which is identical to the well-known and exact Full Configuration Interaction (FCI) method.

However, when deriving the theory of coupled cluster gradients and properties, it is more convenient to use the stationary coupled cluster energy functional, $\underline{\underline{41}}$

$$
\begin{aligned}
E_{C C} & =\langle 0|(1+\hat{\Lambda}) \bar{H}| 0\rangle \\
\hat{\Lambda}=\sum_{k=1}^{N} \hat{\Lambda}_{k} & =\sum_{k=1}^{N} \frac{1}{(k !)^{2}} \sum_{\substack{a_{1} \ldots a_{k} \\
i_{1} \ldots i_{k}}} \lambda_{a_{1} \ldots a_{k}}^{i_{1} \ldots i_{k}} i_{1}^{\dagger} \ldots i_{k}^{\dagger} a_{k} \ldots a_{1}
\end{aligned}
$$

Since the coupled cluster equations are satisfied,

$$
0=\langle P|\bar{H}| 0\rangle
$$

for all excited determinants $\langle P|=\langle S|+\langle D|+\langle T|+\langle Q|+\ldots$ (i.e. single, double, triple, quadruples excitations etc.) up to the number of excitations included in the model, then the energy obtained with this functional is trivially the same as in (1). For CCSDT and $\operatorname{CCSDT}(\mathrm{Q}),\langle P|=\langle S|+\langle D|+\langle T|$. However, both derivatives of the energy with respect to some parameter $\chi$ and expectation values of an arbitrary property $\hat{O}$ can be succinctly written using this same functional,

$$
\begin{aligned}
\frac{\partial E_{C C}}{\partial \chi}=E_{C C}^{\chi} & =\left\langle 0\left|(1+\hat{\Lambda}) \bar{H}^{\chi}\right| 0\right\rangle \\
\langle\hat{O}\rangle_{C C} & =\langle 0|(1+\hat{\Lambda}) \bar{O}| 0\rangle
\end{aligned}
$$

where $\bar{H}^{\chi}=\left(\hat{H}^{\chi} e^{\hat{T}}\right)_{c}=\left(\left(\frac{\partial \hat{H}}{\partial \chi}\right) e^{\hat{T}}\right)_{c}$ and $\bar{O}=\left(\hat{O} e^{\hat{T}}\right)_{c}$. These expressions can be further generalized by constructing one- and two-particle density matrices,

$$
E_{C C}^{\chi}=\sum_{p q} D_{q}^{p}\left(f_{q}^{p}\right)^{\chi}+\sum_{p q r s} \Gamma_{r s}^{p q}\left(v_{r s}^{p q}\right)^{\chi}
$$




$$
\begin{aligned}
\langle\hat{O}\rangle_{C C} & =\sum_{p q} D_{q}^{p} o_{q}^{p}+\sum_{p q r s} \Gamma_{r s}^{p q} o_{r s}^{p q} \\
D_{q}^{p} & =\left\langle 0\left|(1+\hat{\Lambda})\left(\left\{p^{\dagger} q\right\} e^{\hat{T}}\right)_{c}\right| 0\right\rangle \\
\Gamma_{r s}^{p q} & =\left\langle 0\left|(1+\hat{\Lambda})\left(\left\{p^{\dagger} q^{\dagger} s r\right\} e^{\hat{T}}\right)_{c}\right| 0\right\rangle
\end{aligned}
$$

assuming that the operator $\hat{O}$ may have one-electron and/or two-electron components.

The partial derivatives in (9) and (10) are derivatives of the molecular orbital basis quantities, and include contributions from the response of the orbitals to the perturbation. $\underline{41}, 47$ These contributions can be separated out and explicit dependence on the perturbation removed through the use of the coupled perturbed Hartree Fock (CPHF) $Z$-vector equations (similarly to how the $\hat{\Lambda}$ operator removes the dependence on the differentiated $\hat{T}$ amplitudes). $\underline{\underline{32}}$ The relationship of the orbital-response-corrected density to the molecular orbital density is entirely independent of the source of the density, and so is not specific to the particular flavor of coupled cluster theory or even to coupled cluster at all. Similarly, as many coupled cluster methods, including $\operatorname{CCSDT}(\mathrm{Q})$, require semi-canonical orbitals (i.e. $f_{b}^{a}=\epsilon_{a} \delta_{a b}$ and $f_{j}^{i}=\epsilon_{i} \delta_{i j}$ ), the modification of the density matrix to the use of perturbed canonical orbitals is also independent of the source of the density matrix. $\cdot \underline{39} \underline{40}$ This means that for methods which require (semi-)canonical orbitals, only the diagonal elements of the virtual-virtual and occupied-occupied one-electron density matrices need to be computed.

\subsection{The CCSDT(Q) Energy}

The derivation of the CCSDT(Q) energy is similar in many ways to the derivation of the $\operatorname{CCSD}(\mathrm{T})$ energy. In the context of many-body perturbation theory (MBPT), taking the lowest-order correction to the CCSDT energy, and replacing the approximate $\hat{T}_{1}, \hat{T}_{2}$, and $\hat{T}_{3}$ amplitudes with their converged CCSDT values gives the CCSDT[Q] method of Kucharski and Bartlett, $\underline{\underline{48}}$

$$
E_{[Q]}=\left\langle 0\left|\hat{T}_{2}^{\dagger} \hat{V} \hat{R}_{4}\left(\hat{V}\left(\hat{T}_{3}+\frac{1}{2} \hat{T}_{2}^{2}\right)\right)_{c}\right| 0\right\rangle
$$


where $\hat{R}_{4}$ is the resolvent operator in the quadruples space, $\hat{R}_{4}=|Q\rangle\langle Q|\hat{F}| Q\rangle^{-1}\langle Q|$. This approach is similar to that employed in the CCSD[T] (also called CCSD $+\mathrm{T}(\mathrm{CCSD})$ ) method. However, much as $\operatorname{CCSD}(\mathrm{T})$ significantly improves on $\operatorname{CCSD}[\mathrm{T}]$ through the inclusion of a single higher-order term, CCSDT[Q] can be improved upon.

Following the analogue of the derivation of $\operatorname{CCSD}(\mathrm{T})$ by Stanton $\underline{\underline{49}}$ (as done by Bomble et al. $\underline{\underline{23}}$ and Kállay et al. $\underline{\underline{34}}$ ), the exact energy $E$ can be written by applying the Löwdin partitioning approach and then expanded in perturbation orders along with the transformed Hamiltonian,

$$
\begin{aligned}
E & =\left\langle 0\left|\left(1+\hat{\Lambda}_{C C S D T}\right) \bar{H}_{C C S D T}\right| \tilde{P}\right\rangle\left\langle\tilde{P}\left|\left(\bar{H}_{C C S D T}-E\right)^{-1}\right| \tilde{P}\right\rangle\left\langle\tilde{P}\left|\bar{H}_{C C S D T}\right| 0\right\rangle \\
E & =E^{[0]}+E^{[1]}+E^{[2]}+\ldots \\
\bar{H}_{C C S D T} & =\bar{H}_{C C S D T}^{[0]}+\bar{H}_{C C S D T}^{[1]}+\bar{H}_{C C S D T}^{[2]}+\ldots
\end{aligned}
$$

where $\hat{\Lambda}_{C C S D T}=\hat{\Lambda}_{1}+\hat{\Lambda}_{2}+\hat{\Lambda}_{3}, \bar{H}_{C C S D T}=\left(\hat{H} e^{\hat{T}_{1}+\hat{T}_{2}+\hat{T}_{3}}\right)_{c}$, and $|\tilde{P}\rangle=|1-P\rangle=|Q\rangle+\ldots$ is the complement space. Perturbation orders are assigned according to the usual MøllerPlesset partitioning. Taking the lowest (fourth)-order contribution gives the $\operatorname{CCSDT}(\mathrm{Q})_{\Lambda}$ (also called $\Lambda$ CCSDT $(\mathrm{Q})$ ) energy,

$$
\begin{aligned}
E_{(Q)_{\Lambda}} & =\left\langle 0\left|\left(1+\hat{\Lambda}_{C C S D T}\right) \bar{H}_{C C S D T}^{[1]}\right| Q\right\rangle\left\langle Q\left|\left(\bar{H}_{C C S D T}^{[0]}-E^{[0]}\right)^{-1}\right| Q\right\rangle\left\langle Q\left|\bar{H}_{C C S D T}^{[3]}\right| 0\right\rangle \\
& =\left\langle 0\left|\left(\hat{\Lambda}_{2}+\hat{\Lambda}_{3}\right) \hat{V} \hat{T}_{4}^{[3]}\right| 0\right\rangle \\
\hat{T}_{4}^{[3]} & =\hat{R}_{4}\left(\hat{V}\left(\hat{T}_{3}+\frac{1}{2} \hat{T}_{2}^{2}\right)\right)_{c}
\end{aligned}
$$

For canonical Hartree Fock references (or other references where $f_{i}^{a}=f_{a}^{i}=0$ ), the converged $\hat{\Lambda}_{2}$ and $\hat{\Lambda}_{3}$ amplitudes are the same as $\hat{T}_{2}$ and $\hat{T}_{3}$ to lowest order. Thus, as in $\operatorname{CCSD}(\mathrm{T})$, the $\hat{\Lambda}$ amplitudes may be approximated by $\hat{T}^{\dagger}$, giving the CCSDT(Q) method,

$$
E_{(Q)}=\left\langle 0\left|\left(\hat{T}_{2}^{\dagger}+\hat{T}_{3}^{\dagger}\right) \hat{V} \hat{T}_{4}^{[3]}\right| 0\right\rangle
$$


For non-Hartree Fock references such as ROHF and QRHF, though, Kállay notes that $\hat{T}_{3}$ and $\hat{\Lambda}_{3}$ are no longer identical at lowest order. $\underline{\underline{33}}$ By incorporating the additional lowestorder (disconnected) contributions and replacing approximate $\hat{T}_{1}$ and $\hat{T}_{2}$ amplitudes with their converged counterparts at one of two stages, two additional methods, CCSDT(Q)/A and $\operatorname{CCSDT}(\mathrm{Q}) / \mathrm{B}$ may be derived,

$$
\begin{aligned}
E_{(Q) / A} & =\left\langle 0\left|\left(\hat{T}_{2}^{\dagger}+\hat{T}_{3}^{\dagger}+\hat{T}_{1}^{\dagger} \hat{T}_{2}^{\dagger}\right) \hat{H} \hat{T}_{4}^{[3]}\right| 0\right\rangle \\
E_{(Q) / B} & =\left\langle 0\left|\left(\hat{T}_{2}^{\dagger}+\hat{T}_{3}^{\dagger}+\left(\hat{T}_{1}^{\dagger} \hat{V}+\hat{T}_{2}^{\dagger} \hat{F}\right) \hat{R}_{3}\right) \hat{H} \hat{T}_{4}^{[3]}\right| 0\right\rangle \\
& =\left\langle 0\left|\left(\hat{T}_{2}^{\dagger}+\hat{T}_{3}^{\dagger}+\hat{T}_{3}^{B}\right) \hat{H} \hat{T}_{4}^{[3]}\right| 0\right\rangle
\end{aligned}
$$

One difficulty with these methods, though, is that when applied to a canonical reference, they do not reduce to the "normal" $\operatorname{CCSDT}(\mathrm{Q})$ energy. While the additional contributions to $\hat{\Lambda}_{3}$ in the non-HF case are technically at the same order as the portion in common with $\hat{T}_{3}$, these terms are entirely disconnected, which tends to produce a numerically less significant contribution. On the other hand, the balance between connected and disconnected terms is sometimes necessary to avoid excessive basis set dependency and other problems, as in the case of CCSDT[Q] vs. CCSDT(Q). The gradients of the normal HF CCSDT(Q) method as well as the two non-HF variants will be examined on an equal footing.

\subsection{CCSDT(Q) Gradients}

The gradient of the CCSDT(Q) energy can be simply obtained by differentiating the energy expression:

$$
\begin{aligned}
E_{(Q)}^{\chi}= & \left\langle 0\left|\left(\hat{T}_{2}^{\chi \dagger}+\hat{T}_{3}^{\chi \dagger}\right) \hat{V} \hat{T}_{4}^{[3]}\right| 0\right\rangle+\left\langle 0\left|\left(\hat{T}_{2}^{\dagger}+\hat{T}_{3}^{\dagger}\right) \hat{V}^{\chi} \hat{T}_{4}^{[3]}\right| 0\right\rangle-\left\langle 0\left|\hat{T}_{4}^{\prime} \hat{F}^{\chi} \hat{T}_{4}^{[3]}\right| 0\right\rangle \\
& +\left\langle 0\left|\hat{T}_{4}^{\prime}\left(\hat{V}^{\chi}\left(\hat{T}_{3}+\frac{1}{2} \hat{T}_{2}^{2}\right)\right)_{c}\right| 0\right\rangle+\left\langle 0\left|\hat{T}_{4}^{\prime}\left(\hat{V}\left(\hat{T}_{3}^{\chi}+\hat{T}_{2} \hat{T}_{2}^{\chi}\right)\right)_{c}\right| 0\right\rangle \\
E_{(Q) / A}^{\chi}= & \left\langle 0\left|\left(\hat{T}_{2}^{\chi \dagger}+\hat{T}_{3}^{\chi \dagger}+\hat{T}_{1}^{\chi \dagger} \hat{T}_{2}^{\dagger}+\hat{T}_{1} \hat{T}_{2}^{\chi \dagger}\right) \hat{H} \hat{T}_{4}^{[3]}\right| 0\right\rangle+\left\langle 0\left|\left(\hat{T}_{2}^{\dagger}+\hat{T}_{3}^{\dagger}+\hat{T}_{1}^{\dagger} \hat{T}_{2}^{\dagger}\right) \hat{H}^{\chi} \hat{T}_{4}^{[3]}\right| 0\right\rangle
\end{aligned}
$$




$$
\begin{aligned}
& -\left\langle 0\left|\hat{T}_{4}^{A} \hat{F}^{\chi} \hat{T}_{4}^{[3]}\right| 0\right\rangle+\left\langle 0\left|\hat{T}_{4}^{A}\left(\hat{V}^{\chi}\left(\hat{T}_{3}+\frac{1}{2} \hat{T}_{2}^{2}\right)\right)_{c}\right| 0\right\rangle \\
& +\left\langle 0\left|\hat{T}_{4}^{A}\left(\hat{V}\left(\hat{T}_{3}^{\chi}+\hat{T}_{2} \hat{T}_{2}^{\chi}\right)\right)_{c}\right| 0\right\rangle \\
E_{(Q) / B}^{\chi}= & \left\langle 0\left|\left(\hat{T}_{2}^{\chi \dagger}+\hat{T}_{3}^{\chi \dagger}+\left(\hat{T}_{1}^{\chi \dagger} \hat{V}+\hat{T}_{2}^{\chi \dagger} \hat{F}\right) \hat{R}_{3}\right) \hat{H} \hat{T}_{4}^{[3]}\right| 0\right\rangle \\
& +\left\langle 0\left|\left(\hat{T}_{1}^{\dagger} \hat{V}^{\chi}+\hat{T}_{2}^{\dagger} \hat{F}^{\chi}\right) \hat{R}_{3} \hat{H}^{[3]}\right| 0\right\rangle-\left\langle 0\left|\hat{T}_{3}^{B} \hat{F}^{\chi} \hat{R}_{3} \hat{H} \hat{T}_{4}^{[3]}\right| 0\right\rangle \\
& +\left\langle 0\left|\left(\hat{T}_{2}^{\dagger}+\hat{T}_{3}^{\dagger}+\hat{T}_{3}^{B}\right) \hat{H}^{\chi} \hat{T}_{4}^{[3]}\right| 0\right\rangle-\left\langle 0\left|\hat{T}_{4}^{B} \hat{F}^{\chi} \hat{T}_{4}^{[3]}\right| 0\right\rangle \\
& +\left\langle 0\left|\hat{T}_{4}^{B}\left(\hat{V}^{\chi}\left(\hat{T}_{3}+\frac{1}{2} \hat{T}_{2}^{2}\right)\right)_{c}\right| 0\right\rangle+\left\langle 0\left|\hat{T}_{4}^{B} \hat{R}_{4}\left(\hat{V}\left(\hat{T}_{3}^{\chi}+\hat{T}_{2} \hat{T}_{2}^{\chi}\right)\right)_{c}\right| 0\right\rangle
\end{aligned}
$$

where we have defined convenient "left-hand" $\hat{T}_{4}$ intermediates,

$$
\begin{aligned}
& \hat{T}_{4}^{\prime}=\left(\hat{T}_{2}^{\dagger}+\hat{T}_{3}^{\dagger}\right) \hat{V} \hat{R}_{4} \\
& \hat{T}_{4}^{A}=\left(\hat{T}_{2}^{\dagger}+\hat{T}_{3}^{\dagger}+\hat{T}_{1}^{\dagger} \hat{T}_{2}^{\dagger}\right) \hat{H} \hat{R}_{4} \\
& \hat{T}_{4}^{B}=\left(\hat{T}_{2}^{\dagger}+\hat{T}_{3}^{\dagger}+\hat{T}_{3}^{B}\right) \hat{H} \hat{R}_{4}
\end{aligned}
$$

and used the identity $\hat{R}_{n}^{\chi}=-\hat{R}_{n} \hat{F}^{\chi} \hat{R}_{n}$. We can separate each of these expressions into two parts: one which depends on derivatives of the Hamiltonian multiplied by CCSDT(Q) density matrix elements, and one which depends on derivatives of the coupled cluster amplitudes:

$$
\begin{aligned}
E_{(Q)}^{\chi}= & \left\langle 0\left|D_{(Q)}^{\prime} \hat{F}^{\chi}\right| 0\right\rangle+\left\langle 0\left|\Gamma_{(Q)}^{\prime} \hat{V}^{\chi}\right| 0\right\rangle+\left\langle 0\left|\hat{S}_{2} \hat{T}_{2}^{\chi}\right| 0\right\rangle+\left\langle 0\left|\hat{S}_{3} \hat{T}_{3}^{\chi}\right| 0\right\rangle \\
E_{(Q) / A, B}^{\chi}= & \left\langle 0\left|D_{(Q) / A, B}^{\prime} \hat{F}^{\chi}\right| 0\right\rangle+\left\langle 0\left|\Gamma_{(Q) / A, B}^{\prime} \hat{V}^{\chi}\right| 0\right\rangle+\left\langle 0\left|\hat{S}_{1}^{A / B} \hat{T}_{1}^{\chi}\right| 0\right\rangle \\
& +\left\langle 0\left|\hat{S}_{2}^{A / B} \hat{T}_{2}^{\chi}\right| 0\right\rangle+\left\langle 0\left|\hat{S}_{3}^{A / B} \hat{T}_{3}^{\chi}\right| 0\right\rangle
\end{aligned}
$$

given in terms of the density matrices,

$$
\begin{aligned}
\left(D_{(Q)}^{\prime}\right)_{q}^{p} & =-\delta_{p q}\left\langle 0\left|\hat{T}_{4}^{\prime}\left\{p^{\dagger} q\right\} \hat{T}_{4}^{[3]}\right| 0\right\rangle \\
\left(D_{(Q) / A}^{\prime}\right)_{q}^{p} & =\Delta_{p q}\left\langle 0\left|\left(\hat{T}_{3}^{\dagger}+\hat{T}_{1}^{\dagger} \hat{T}_{2}^{\dagger}-\hat{T}_{4}^{A}\right)\left\{p^{\dagger} q\right\} \hat{T}_{4}^{[3]}\right| 0\right\rangle \\
\left(D_{(Q) / B}^{\prime}\right)_{q}^{p} & =\Delta_{p q}\left\{\left\langle 0\left|\left(\hat{T}_{3}^{\dagger}+\hat{T}_{3}^{B}-\hat{T}_{4}^{B}\right)\left\{p^{\dagger} q\right\} \hat{T}_{4}^{[3]}\right| 0\right\rangle\right.
\end{aligned}
$$




$$
\begin{aligned}
& \left.+\left\langle 0\left|\left(\hat{T}_{2}^{\dagger}-\hat{T}_{3}^{B}\right)\left\{p^{\dagger} q\right\} \hat{R}_{3} \hat{H} \hat{T}_{4}^{[3]}\right| 0\right\rangle\right\} \\
\left(\Gamma_{(Q)}^{\prime}\right)_{r s}^{p q}= & \left\langle 0\left|\left(\hat{T}_{2}^{\dagger}+\hat{T}_{3}^{\dagger}\right)\left\{p^{\dagger} q^{+} s r\right\} \hat{T}_{4}^{[3]}\right| 0\right\rangle+\left\langle 0\left|\hat{T}_{4}^{\prime}\left(\left\{p^{\dagger} q^{\dagger} s r\right\}\left(\hat{T}_{3}+\frac{1}{2} \hat{T}_{2}^{2}\right)\right)_{c}\right| 0\right\rangle \\
\left(\Gamma_{(Q) / A}^{\prime}\right)_{r s}^{p q}= & \left\langle 0\left|\left(\hat{T}_{2}^{\dagger}+\hat{T}_{3}^{\dagger}+\hat{T}_{1}^{\dagger} \hat{T}_{2}^{\dagger}\right)\left\{p^{\dagger} q^{\dagger} s r\right\} \hat{T}_{4}^{[3]}\right| 0\right\rangle \\
& +\left\langle 0\left|\hat{T}_{4}^{A}\left(\left\{p^{\dagger} q^{\dagger} s r\right\}\left(\hat{T}_{3}+\frac{1}{2} \hat{T}_{2}^{2}\right)\right)_{c}\right| 0\right\rangle \\
\left(\Gamma_{(Q) / B}^{\prime}\right)_{r s}^{p q}= & \left\langle 0\left|\left(\hat{T}_{2}^{\dagger}+\hat{T}_{3}^{\dagger}+\hat{T}_{3}^{B}\right)\left\{p^{\dagger} q^{\dagger} s r\right\} \hat{T}_{4}^{[3]}\right| 0\right\rangle+\left\langle 0\left|\hat{T}_{1}^{\dagger}\left\{p^{\dagger} q^{\dagger} s r\right\} \hat{R}_{3} \hat{H} \hat{T}_{4}^{[3]}\right| 0\right\rangle \\
& +\left\langle 0\left|\hat{T}_{4}^{B}\left(\left\{p^{\dagger} q^{\dagger} s r\right\}\left(\hat{T}_{3}+\frac{1}{2} \hat{T}_{2}^{2}\right)\right)_{c}\right| 0\right\rangle
\end{aligned}
$$

where $\Delta_{p q}=\delta_{p q}+\delta_{p a} \delta_{q i}+\delta_{p i} \delta_{q a}$, and intermediates,

$$
\begin{aligned}
& \hat{S}_{1}^{A}=\hat{T}_{4}^{[3] \dagger} \hat{H} \hat{T}_{2}|S\rangle\langle S| \\
& \hat{S}_{1}^{B}=\hat{T}_{4}^{[3] \dagger} \hat{H} \hat{R}_{3} \hat{V}|S\rangle\langle S| \\
& \hat{S}_{2}=\left(\hat{T}_{4}^{[3] \dagger} \hat{V}+\hat{T}_{4}^{\prime}\left(\hat{\left.\left.\hat{V} \hat{T}_{2}\right)_{c}\right)|D\rangle}\langle D|\right.\right. \\
& \hat{S}_{2}^{A}=\left(\hat{T}_{4}^{[3] \dagger}\left(\hat{V}+\hat{H} \hat{T}_{1}\right)+\hat{T}_{4}^{A}\left(\hat{\left.\left.\hat{V} \hat{T}_{2}\right)_{c}\right)|D\rangle}\langle D|\right.\right.
\end{aligned}
$$

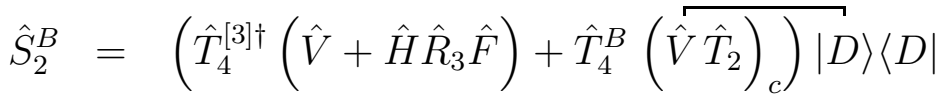

$$
\begin{aligned}
& \hat{S}_{3}=\left(\hat{T}_{4}^{[3] \dagger}+\hat{T}_{4}^{\prime}\right) \hat{V}|T\rangle\langle T| \\
& \hat{S}_{3}^{A, B}=\left(\hat{T}_{4}^{[3] \dagger} \hat{H}+\hat{T}_{4}^{A, B} \hat{V}\right)|T\rangle\langle T|
\end{aligned}
$$

where the contraction line indicates that at least one creation/annihilation operator in $\hat{V}$ must remain uncontracted (i.e. $\hat{V}$ must have at least one external line in the diagrammatic representation). The factor $\Delta_{p q}$ indicates that the density matrix elements only need be computed for the occupied-virtual and virtual-occupied blocks and for the diagonal of the occupied-occupied and virtual-virtual blocks. Note that the CCSDT(Q) one-particle density matrix does not have an occupied-virtual contribution, and is only valid for canonical (HF) references.

At this point the equations for the $\operatorname{CCSDT}(\mathrm{Q})$ and $\operatorname{CCSDT}(\mathrm{Q}) / \mathrm{A}, \mathrm{B}$ gradients may be 
combined in a generic expression,

$$
E_{(Q) / X}^{\chi}=\left\langle 0\left|D_{(Q) / X}^{\prime} \hat{F}^{\chi}\right| 0\right\rangle+\left\langle 0\left|\Gamma_{(Q) / X}^{\prime} \hat{V}^{\chi}\right| 0\right\rangle+\left\langle 0\left|\hat{S}^{X} \hat{T}^{\chi}\right| 0\right\rangle
$$

where $\hat{S}^{X}=\hat{S}_{1}^{X}+\hat{S}_{2}^{X}+\hat{S}_{3}^{X}$ and either $X=A, B$ or is "empty", indicating the CCSDT(Q) gradient and intermediates (with $\hat{S}_{1}=0$ ). Now, the definition of the derivative coupled cluster amplitudes (obtained by differentiating the coupled cluster amplitude equations) may be inserted to transform dependence on the derivative amplitudes to dependence on the derivative Hamiltonian,

$$
\begin{aligned}
E_{(Q) / X}^{\chi} & =\left\langle 0\left|D_{(Q) / X}^{\prime} \hat{F}^{\chi}\right| 0\right\rangle+\left\langle 0\left|\Gamma_{(Q) / X}^{\prime} \hat{V}^{\chi}\right| 0\right\rangle-\left\langle 0\left|\hat{S}^{X}\left(\bar{H}-E_{C C S D T}\right)^{-1}\right| P\right\rangle\left\langle P\left|\bar{H}^{\chi}\right| 0\right\rangle \\
& =\left\langle 0\left|D_{(Q) / X}^{\prime} \hat{F}^{\chi}\right| 0\right\rangle+\left\langle 0\left|\Gamma_{(Q) / X}^{\prime} \hat{V}^{\chi}\right| 0\right\rangle+\left\langle 0\left|\tilde{\Lambda}^{X} \bar{H}^{\chi}\right| 0\right\rangle
\end{aligned}
$$

Lastly, the solution of the $\tilde{\Lambda}^{X}$ equations can be combined with the solution of the CCSDT $\hat{\Lambda}$ equations by solving for their linear combination,

$$
\left\langle 0\left|\left(\hat{\Lambda}+\tilde{\Lambda}^{X}\right)\left(\bar{H}-E_{C C S D T}\right)\right| P\right\rangle+\left\langle 0\left|\left(\bar{H}+\hat{S}^{X}\right)\right| P\right\rangle=0
$$

and the combined density matrices constructed which give the gradient of the total CCSDT(Q)/X energy (total correlation energy; the gradient of the reference energy is computed and added in the standard way),

$$
\begin{aligned}
E_{C C S D T(Q) / X}^{\chi} & =\left\langle 0\left|D_{C C S D T(Q) / X} \hat{F}^{\chi}\right| 0\right\rangle+\left\langle 0\left|\Gamma_{C C S D T(Q) / X} \hat{V}^{\chi}\right| 0\right\rangle \\
\left(D_{C C S D T(Q) / X}\right)_{q}^{p} & =\left(D_{(Q) / X}^{\prime}\right)_{q}^{p}+\left\langle 0\left|\left(1+\hat{\Lambda}+\tilde{\Lambda}^{X}\right)\left(\left\{p^{\dagger} q\right\} e^{\hat{T}}\right)_{c}\right| 0\right\rangle \\
\left(\Gamma_{C C S D T(Q) / X}\right)_{r s}^{p q} & =\left(\Gamma_{(Q) / X}^{\prime}\right)_{r s}^{p q}+\left\langle 0\left|\left(1+\hat{\Lambda}+\tilde{\Lambda}^{X}\right)\left(\left\{p^{\dagger} q^{\dagger} s r\right\} e^{\hat{T}}\right)_{c}\right| 0\right\rangle
\end{aligned}
$$

These density matrices may then be processed in the usual way to compute energy gradients and properties as desired. 


\subsection{Iterative Approximations}

The CCSDT(Q) energy is a non-iterative correction that is applied after the CCSDT equations have converged. Another approach to approximating the CCSDTQ energy may be obtained by deleting some terms from the CCSDTQ equations, but maintaining the iterative structure of the problem. In particular, we wish to delete at least all $\hat{T}_{4} \rightarrow \hat{T}_{4}$ terms

except those coming from $\hat{H}^{[0]} \equiv \sum_{p} f_{p}^{p}\left\{p^{\dagger} p\right\}$. This leads to an equation for the $\hat{T}_{4}$ amplitudes of the form $0=\left\langle Q\left|\tilde{H}+\hat{H}^{[0]} \hat{T}_{4}\right| 0\right\rangle$ or equivalently $\hat{T}_{4}=\hat{R}_{4}\langle Q|\tilde{H}| 0\rangle$, where $\tilde{H}$ is an effective operator discussed below. Because the $\hat{T}_{4}$ equations can be solved exactly (given $\hat{T}_{1}-\hat{T}_{3}$ amplitudes which may solve their own equations only approximately), the $\hat{T}_{4}$ amplitudes will only appear as "intermediates"- directly constructed from $\hat{T}_{1}, \hat{T}_{2}$, and $\hat{T}_{3}$ and then immediately consumed in the remaining amplitudes equations. This structure both eliminates the costly $\mathscr{O}\left(n^{10}\right)$ steps of the CCSDTQ equations and allows for reduced storage of $\hat{T}_{4}$ since it may be immediately calculated, used, and discarded.

Depending on the additional terms that are deleted (guided by a mixture of perturbation theory and pragmatism), the following approximations may derived, $\underline{\underline{34}}$

CCSDTQ-1a ( CCSDTQ-1):

$$
\begin{aligned}
\hat{T}_{4}^{C C S D T Q-1 a} & =\hat{R}_{4}\left(\hat{V}\left(\hat{T}_{3}+\frac{1}{2} \hat{T}_{2}^{2}\right)\right)_{c}=\hat{T}_{4}^{[3]} \\
0 & =\left\langle S\left|\bar{H}_{C C S D T}\right| 0\right\rangle \\
0 & \left.=\langle D| \bar{H}_{C C S D T}+\hat{V} \hat{T}_{4}^{C C S D T Q-1 a}\right]|0\rangle \\
0 & =\left\langle T\left|\bar{H}_{C C S D T}\right| 0\right\rangle
\end{aligned}
$$

CCSDTQ-1b:

$$
\begin{aligned}
\hat{T}_{4}^{C C S D T Q-1 b} & =\hat{R}_{4}\left(\hat{V}\left(\hat{T}_{3}+\frac{1}{2} \hat{T}_{2}^{2}\right)\right)_{c} \\
0 & =\left\langle S\left|\bar{H}_{C C S D T}\right| 0\right\rangle
\end{aligned}
$$




$$
\begin{aligned}
& \left.0=\langle D| \bar{H}_{C C S D T}+\hat{V} \hat{T}_{4}^{C C S D T Q-1 b}\right]|0\rangle \\
& 0=\left\langle T\left|\bar{H}_{C C S D T}+\left(\left(\hat{H}+\hat{V} \hat{T}_{1}\right) \hat{T}_{4}^{C C S D T Q-1 b}\right)_{c}\right| 0\right\rangle
\end{aligned}
$$

CCSDTQ-3:

$$
\begin{aligned}
\hat{T}_{4}^{C C S D T Q-3} & =\hat{R}_{4}\left(\hat{H} e^{\hat{T}_{1}+\hat{T}_{2}}\left(1+\hat{T}_{3}\right)+\left(\hat{P}_{T} \hat{V} \hat{T}_{3} \hat{P}_{D}\right) \hat{T}_{3}\right)_{c} \\
0 & =\left\langle S\left|\bar{H}_{C C S D T}\right| 0\right\rangle \\
0 & \left.=\langle D| \bar{H}_{C C S D T}+\hat{V} \hat{T}_{4}^{C C S D T Q-3}\right]|0\rangle \\
0 & =\left\langle T\left|\bar{H}_{C C S D T}+\left(\left(\hat{H}+\hat{V} \hat{T}_{1}\right) \hat{T}_{4}^{C C S D T Q-3}\right)_{c}\right| 0\right\rangle
\end{aligned}
$$

CC4:

$$
\begin{aligned}
\hat{T}_{4}^{C C 4} & =\hat{R}_{4}\left(\hat{V}^{\prime}\left(\hat{T}_{3}+\frac{1}{2} \hat{T}_{2}^{2}\right)\right) \\
0 & =\left\langle S\left|\bar{H}_{C C S D T}\right| 0\right\rangle \\
0 & \left.=\langle D| \bar{H}_{C C S D T}+\hat{V} \hat{T}_{4}^{C C 4}\right]|0\rangle \\
0 & =\left\langle T\left|\bar{H}_{C C S D T}+\left(\left(\hat{H}+\hat{V} \hat{T}_{1}\right) \hat{T}_{4}^{C C 4}\right)_{c}\right| 0\right\rangle
\end{aligned}
$$

where $\hat{P}_{X}=|X\rangle\langle X|$ is a projection operator onto the given excitation manifold and $\hat{V}^{\prime}=$ $\left(\hat{V} e^{\hat{T}_{1}}\right)_{c}$ is the $\hat{T}_{1}$-transformed two-electron potential. Note that $\hat{T}_{4}^{C C S D T Q-3}$ is almost equal to $\hat{R}_{4}\left\langle Q\left|\bar{H}_{C C S D T}\right| 0\right\rangle$, but that it differs by the two terms $t_{i j k l}^{a b c d} \leftarrow \frac{1}{4} v_{e f}^{m n} t_{i j k}^{e f c} t_{m n l}^{a b d}+\frac{1}{2} v_{e f}^{m n} t_{m i j}^{e a b} t_{n k l}^{f c d}$ which are excluded by $\left(\hat{P}_{T} \hat{V} \hat{T}_{3} \hat{P}_{D}\right) \hat{T}_{3}$ (since the projection operators and connectivity condition require that $\hat{V}$ be connected to the inner $\hat{T}_{3}$ vertex by exactly three indices). These terms are specifically deleted as they would require $\mathscr{O}\left(n^{10}\right)$ computation.

\subsection{Gradients in the Iterative Approximation}

The derivation of the gradient of the energy in the iterative approximation closely follows the derivation of gradients for canonical CC methods. However, since the cluster equations 
have been modified to delete specific terms, we express the energy and its derivatives using an effective transformed Hamiltonian $\tilde{H}$ (also called the Jacobian),

$$
\begin{aligned}
& E_{X}=\left\langle 0\left|(1+\hat{\Lambda}) \tilde{H}_{X}\right| 0\right\rangle \\
& E_{X}^{\chi}=\left\langle 0\left|(1+\hat{\Lambda}) \tilde{H}_{X}^{\chi}\right| 0\right\rangle
\end{aligned}
$$

where $X$ is one of the approximate methods above. The effective transformed Hamiltonian may be specified in block form for each of the approximate methods,

$$
\tilde{H}_{X}=\left(\begin{array}{ccccc}
0 & S & D & T & Q \\
E_{X} & \bar{H}_{0 S} & \hat{V} & 0 & 0 \\
\tilde{H}_{S 0} & \bar{H}_{S S} & \bar{H}_{S D} & \hat{V} & 0 \\
\tilde{H}_{D 0} & \bar{H}_{D S} & \bar{H}_{D D} & \bar{H}_{D T} & \hat{V} \\
\tilde{H}_{T 0} & \bar{H}_{T S} & \bar{H}_{T D} & \bar{H}_{T T} & \tilde{H}_{T Q} \\
\tilde{H}_{Q 0} & \tilde{H}_{Q S} & \tilde{H}_{Q D} & \tilde{H}_{Q T} & \hat{H}^{[0]}
\end{array}\right)\left(\begin{array}{c}
T \\
Q
\end{array}\right.
$$

The $\bar{H}$ blocks are identical to the corresponding blocks of $\bar{H}_{C C S D T} . \tilde{H}_{T Q}$ is zero for CCSDTQ1a and equal to $\hat{H}+\left[\hat{V}, \hat{T}_{1}\right]$ for all other methods. The remaining blocks may be derived for each method from the corresponding equation for $\hat{T}_{4}$, such that $\hat{T}_{4}=\hat{R}_{4} \tilde{H}_{Q 0}, \frac{\partial \hat{T}_{4}}{\partial \tilde{T}_{1}}=\hat{R}_{4} \tilde{H}_{Q S}$, and similarly for $\tilde{H}_{Q D}$ and $\tilde{H}_{Q T}$, and from the modified amplitude equations, $\tilde{H}_{S 0}=\tilde{H}_{D 0}=$ $\tilde{H}_{T 0}=0$.

The stationarity conditions of the energy functional ([66) and (67)) along with the definition of $\tilde{H}$ determine the equations for $\hat{\Lambda}$,

CCSDTQ-1a:

$$
\begin{aligned}
\hat{\Lambda}_{4}^{C C S D T Q-1 a} & =\hat{\Lambda}_{2} \hat{V} \hat{R}_{4}=\hat{\Lambda}_{4}^{[2]} \\
0 & =\langle 0 \mid(1+\hat{\Lambda}) \overline{\bar{H} \mid S}\rangle_{C C S D T} \\
0 & =\langle 0 \mid(1+\hat{\Lambda}) \overline{\bar{H} \mid D}\rangle_{C C S D T}+\left\langle 0\left|\hat{\Lambda}_{4}^{C C S D T Q-1 a}\left[\hat{\bar{V}}, \hat{T}_{2}\right]\right| D\right\rangle
\end{aligned}
$$




$$
0=\langle 0 \mid(1+\hat{\Lambda}) \overline{\bar{H} \mid T}\rangle_{C C S D T}+\left\langle 0\left|\hat{\Lambda}_{4}^{C C S D T Q-1 a} \hat{V}\right| T\right\rangle
$$

CCSDTQ-1b:

$$
\begin{aligned}
\hat{\Lambda}_{4}^{C C S D T Q-1 b} & =\left(\hat{\Lambda}_{2} \hat{V}+\hat{\Lambda}_{3}\left(\hat{H}+\left[\hat{V}, \hat{T}_{1}\right]\right)\right) \hat{R}_{4} \\
0 & =\langle 0 \mid(1+\hat{\Lambda}) \overline{\bar{H} \mid S}\rangle_{C C S D T}+\langle 0| \hat{\Lambda}_{3} \hat{\hat{V} \hat{T}_{4}^{C C S D T Q-1 b}|S\rangle} \\
0 & =\langle 0 \mid(1+\hat{\Lambda}) \overline{\bar{H} \mid D}\rangle_{C C S D T}+\left\langle 0\left|\hat{\Lambda}_{4}^{C C S D T Q-1 b}\left[\hat{V}, \hat{T_{2}}\right]\right| D\right\rangle \\
0 & =\langle 0 \mid(1+\hat{\Lambda}) \overline{\bar{H} \mid T}\rangle_{C C S D T}+\left\langle 0\left|\hat{\Lambda}_{4}^{C C S D T Q-1 b} \hat{V}\right| T\right\rangle
\end{aligned}
$$

\section{CCSDTQ-3:}

$$
\begin{aligned}
& \hat{\Lambda}_{4}^{C C S D T Q-3}=\left(\hat{\Lambda}_{2} \hat{V}+\hat{\Lambda}_{3}\left(\hat{H}+\left[\hat{V}, \hat{T}_{1}\right]\right)\right) \hat{R}_{4} \\
& 0=\langle 0|(1+\hat{\Lambda}) \bar{H}| S\rangle_{C C S D T}+\langle 0| \hat{\Lambda}_{4}^{C C S D T Q-3}\left(\widehat{\left.\hat{H} e^{\hat{T}_{1}+\hat{T}_{2}}\left(1+\hat{T}_{3}\right)\right)_{c}|S\rangle}\right. \\
& +\left\langle 0\left|\hat{\Lambda}_{3} \hat{V} \hat{T}_{4}^{C C S D T Q-3}\right| S\right\rangle \\
& 0=\langle 0|(1+\hat{\Lambda}) \overline{\bar{H}}| D\rangle_{C C S D T}+\langle 0| \hat{\Lambda}_{4}^{C C S D T Q-3}\left(\hat{\left.\hat{H} e^{\hat{T}_{1}+\hat{T}_{2}}\left(1+\hat{T}_{3}\right)\right)_{c}|D\rangle}\right. \\
& 0=\langle 0 \mid(1+\hat{\Lambda}) \overline{\bar{H} \mid T}\rangle_{C C S D T}+\langle 0| \hat{\Lambda}_{4}^{C C S D T Q-3}\left(\hat{\left.\hat{H} e^{\hat{T}_{1}+\hat{T}_{2}}+\hat{P}_{T} \hat{\hat{V} \hat{T}_{3} \hat{P}_{D}}\right)_{c}|T\rangle}\right. \\
& +\left\langle 0\left|\hat{V}\left(\hat{P}_{D} \hat{\Lambda}_{4}^{C C S D T Q-3} \hat{T}_{3} \hat{P}_{T}\right)\right| T\right\rangle
\end{aligned}
$$

CC4:

$$
\begin{aligned}
\hat{\Lambda}_{4}^{C C 4} & =\left(\hat{\Lambda}_{2} \hat{V}+\hat{\Lambda}_{3}\left(\hat{H}+\left[\hat{V}, \hat{T}_{1}\right]\right)\right) \hat{R}_{4} \\
0 & =\langle 0 \mid(1+\hat{\Lambda}) \overline{\bar{H} \mid S}\rangle_{C C S D T}+\langle 0| \hat{\Lambda}_{4}^{C C 4}\left(\widehat{\left.\hat{V}^{\prime}\left(\hat{T}_{3}+\frac{1}{2} \hat{T}_{2}^{2}\right)\right)_{c}|S\rangle}\right. \\
& +\left\langle 0\left|\hat{\Lambda}_{3} \hat{V} \hat{T}_{4}^{C C 4}\right| S\right\rangle \\
0 & =\langle 0 \mid(1+\hat{\Lambda}) \overline{\bar{H} \mid D}\rangle_{C C S D T}+\langle 0| \hat{\Lambda}_{4}^{C C 4}\left[\overrightarrow{\left.\hat{V}^{\prime}, \hat{T}_{2}\right]|D\rangle}\right. \\
0 & =\langle 0 \mid(1+\hat{\Lambda}) \overline{\bar{H} \mid T}\rangle_{C C S D T}+\left\langle 0\left|\hat{\Lambda}_{4}^{C C 4} \hat{V}^{\prime}\right| T\right\rangle
\end{aligned}
$$




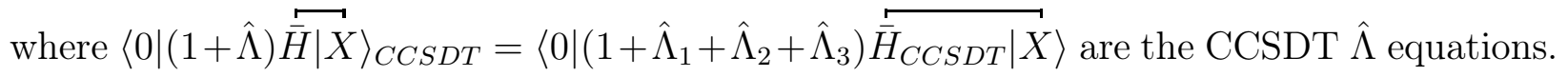
As mentioned by Gauss et al., $\underline{38}$ the equations for the $\hat{\Lambda}$ amplitudes may also be derived diagrammatically by "capping" the each cluster amplitude diagram with the appropriate $\hat{\Lambda}$ amplitude vertex to form a closed diagram, and then sequentially deleting each cluster amplitude vertex to give the set of open $\hat{\Lambda}$ amplitude diagrams.

As with CCSDT(Q), the gradient and molecular properties are computed through the one- and two-particle density matrices. These are easily derived from (67),

$$
\begin{aligned}
& \left(D_{C C S D T Q-1 a}\right)_{q}^{p}=\left(D_{C C S D T}\right)_{q}^{p}-\delta_{p q}\left\langle 0\left|\hat{\Lambda}_{4}^{C C S D T Q-1 a}\left\{p^{\dagger} q\right\} \hat{T}_{4}^{C C S D T Q-1 a}\right| 0\right\rangle \\
& \left(D_{C C S D T Q-1 b}\right)_{q}^{p}=\left(D_{C C S D T}\right)_{q}^{p}+\Delta_{p q}\left\langle 0\left|\left(\hat{\Lambda}_{3}-\hat{\Lambda}_{4}^{C C S D T Q-1 b}\right)\left\{p^{\dagger} q\right\} \hat{T}_{4}^{C C S D T Q-1 b}\right| 0\right\rangle \\
& \left(D_{C C S D T Q-3}\right)_{q}^{p}=\left(D_{C C S D T}\right)_{q}^{p}+\Delta_{p q}\left\langle 0\left|\left(\hat{\Lambda}_{3}-\hat{\Lambda}_{4}^{C C S D T Q-3}\right)\left\{p^{\dagger} q\right\} \hat{T}_{4}^{C C S D T Q-3}\right| 0\right\rangle \\
& +\left\langle 0\left|\hat{\Lambda}_{4}^{C C S D T Q-3}\left(\left\{p^{\dagger} q\right\} \hat{T}_{3} \hat{T}_{2}\right)_{c}\right| 0\right\rangle \\
& \left(D_{C C 4}\right)_{q}^{p}=\left(D_{C C S D T}\right)_{q}^{p}+\Delta_{p q}\left\langle 0\left|\left(\hat{\Lambda}_{3}-\hat{\Lambda}_{4}^{C C 4}\right)\left\{p^{\dagger} q\right\} \hat{T}_{4}^{C C 4}\right| 0\right\rangle \\
& \left(\Gamma_{C C S D T Q-1 a}\right)_{r s}^{p q}=\left(\Gamma_{C C S D T}\right)_{r s}^{p q}+\left\langle 0\left|\hat{\Lambda}_{2}\left\{p^{\dagger} q^{\dagger} s r\right\} \hat{T}_{4}^{C C S D T Q-1 a}\right| 0\right\rangle \\
& +\left\langle 0\left|\hat{\Lambda}_{4}^{C C S D T Q-1 a}\left(\left\{p^{\dagger} q^{\dagger} s r\right\}\left(\hat{T}_{3}+\frac{1}{2} \hat{T}_{2}^{2}\right)\right)_{c}\right| 0\right\rangle \\
& \left(\Gamma_{C C S D T Q-1 b}\right)_{r s}^{p q}=\left(\Gamma_{C C S D T}\right)_{r s}^{p q}+\left\langle 0\left|\hat{\Lambda}_{2}\left\{p^{\dagger} q^{\dagger} s r\right\} \hat{T}_{4}^{C C S D T Q-1 b}\right| 0\right\rangle \\
& +\left\langle 0\left|\hat{\Lambda}_{3}\left(\left\{p^{\dagger} q^{\dagger} s r\right\}\left(1+\hat{T}_{1}\right) \hat{T}_{4}^{C C S D T Q-1 b}\right)_{c}\right| 0\right\rangle \\
& +\left\langle 0\left|\hat{\Lambda}_{4}^{C C S D T Q-1 b}\left(\left\{p^{\dagger} q^{\dagger} s r\right\}\left(\hat{T}_{3}+\frac{1}{2} \hat{T}_{2}^{2}\right)\right)_{c}\right| 0\right\rangle \\
& \left(\Gamma_{C C S D T Q-3}\right)_{r s}^{p q}=\left(\Gamma_{C C S D T}\right)_{r s}^{p q}+\left\langle 0\left|\hat{\Lambda}_{2}\left\{p^{\dagger} q^{\dagger} s r\right\} \hat{T}_{4}^{C C S D T Q-3}\right| 0\right\rangle \\
& +\left\langle 0\left|\hat{\Lambda}_{3}\left(\left\{p^{\dagger} q^{\dagger} s r\right\}\left(1+\hat{T}_{1}\right) \hat{T}_{4}^{C C S D T Q-1 b}\right)_{c}\right| 0\right\rangle \\
& +\left\langle 0\left|\hat{\Lambda}_{4}^{C C S D T Q-1 b}\left(\left\{p^{\dagger} q^{\dagger} s r\right\} e^{\hat{T}_{1}+\hat{T}_{2}}\left(1+\hat{T}_{3}\right)\right)_{c}\right| 0\right\rangle \\
& +\left\langle 0\left|\hat{\Lambda}_{4}^{C C S D T Q-1 b}\left(\left(\hat{P}_{D}\left\{p^{\dagger} q^{\dagger} s r\right\} \hat{T}_{3} \hat{P}_{S}\right) \hat{T}_{3}\right)_{c}\right| 0\right\rangle \\
& \left(\Gamma_{C C 4}\right)_{r s}^{p q}=\left(\Gamma_{C C S D T}\right)_{r s}^{p q}+\left\langle 0\left|\hat{\Lambda}_{2}\left\{p^{\dagger} q^{\dagger} s r\right\} \hat{T}_{4}^{C C 4}\right| 0\right\rangle \\
& +\left\langle 0\left|\hat{\Lambda}_{3}\left(\left\{p^{\dagger} q^{\dagger} s r\right\}\left(1+\hat{T}_{1}\right) \hat{T}_{4}^{C C 4}\right)_{c}\right| 0\right\rangle
\end{aligned}
$$




$$
+\left\langle 0\left|\hat{\Lambda}_{4}^{C C 4}\left(\left\{p^{\dagger} q^{\dagger} s r\right\} e^{\hat{T}_{1}}\left(\hat{T}_{3}+\frac{1}{2} \hat{T}_{2}^{2}\right)\right)_{c}\right| 0\right\rangle
$$

where $\left(D_{C C S D T}\right)_{q}^{p}=\left\langle 0\left|\left(1+\hat{\Lambda}_{1}+\hat{\Lambda}_{2}+\hat{\Lambda}_{3}\right)\left(\left\{p^{\dagger} q\right\} e^{\hat{T}_{1}+\hat{T}_{2}+\hat{T}_{3}}\right)\right| 0\right\rangle$ and $\left(\Gamma_{C C S D T}\right)_{r s}^{p q}=\langle 0|(1+$ $\left.\hat{\Lambda}_{1}+\hat{\Lambda}_{2}+\hat{\Lambda}_{3}\right)\left(\left\{p^{\dagger} q^{\dagger} s r\right\} e^{\hat{T}_{1}+\hat{T}_{2}+\hat{T}_{3}}\right)|0\rangle$ are the CCSDT density matrices, but with cluster and $\hat{\Lambda}$ amplitudes determined by the modified equations for the specific method in question. As with the $\hat{\Lambda}$ amplitudes, the density matrices may be derived diagrammatically starting from the diagrammatic form of the energy functional (66), and then deleting the Hamiltonian vertex. Using the diagrammatic approach, care must be taken with the sign and numerical prefactors such that the definition of the density matrices matches that of (9)).

\section{Implementation}

The total CCSDT(Q), CCSDT(Q)/A, CCSDT(Q)/B, CCSDTQ-1a, CCSDTQ-1b, CCSDTQ3, and CC4 density matrices have been implemented in the NCC module ${ }^{50}$ of the CFOUR program system. ${ }^{51}$ As of this time, this implementation only handles closed-shell reference states, including RHF and closed-shell QRHF (or other restricted non-HF orbitals, e.g. Kohn-Sham orbitals). Additionally, the implementation makes use of non-orthogonal spinadaptation techniques $\underline{50,52}$ as well as recent advances in high-performance tensor contraction, $\underline{53,54}$ which allows for high efficiency and relatively compact working equations.

The cost of a CCSDT(Q) gradient calculation is between two and three times that of the corresponding energy calculation. The energy calculation requires the iterative solution of the CCSDT coupled cluster equations with $\mathscr{O}\left(n^{8}\right)$ cost, and four non-iterative $\mathscr{O}\left(n^{9}\right)$ steps (multiplication of $\hat{T}_{3}$ with $v_{c i}^{a b}$ and $v_{j k}^{i a}$, and $\hat{T}_{2}$ with the three-particle intermediates $\tilde{W}_{i j e}^{a b c}$ and $\tilde{W}_{i j k}^{a b m}$ [see below]). The gradient calculation instead requires two iterative $\mathscr{O}\left(n^{8}\right)$ procedures (the CCSDT coupled cluster equations and the combined $\hat{\Lambda}+\tilde{\Lambda}$ equations) and ten non-iterative $\mathscr{O}\left(n^{9}\right)$ steps. In order to reach this minimal number of $\mathscr{O}\left(n^{9}\right)$ steps, the contributions to $\left(\Gamma_{(Q)}^{\prime}\right)_{c i}^{a b}$ and $\left(\Gamma_{(Q)}^{\prime}\right)_{a b}^{c i}$ must be combined (and similarly for $\left(\Gamma_{(Q)}^{\prime}\right)_{j k}^{i a}$ and 
$\left.\left(\Gamma_{(Q)}^{\prime}\right)_{i a}^{j k}\right)$ when computing the symmetrized two-electron density,

$$
\begin{aligned}
& \left(\Gamma_{(Q)}^{\prime}\right)_{c i}^{a b}=\left(\Gamma_{(Q)}^{\prime}\right)_{a b}^{c i}=\frac{1}{24} \sum_{\text {efmno }}\left\{\left(t^{[3]}\right)_{m n o i}^{e f a b}+\left(t^{\prime}\right)_{m n o i}^{e f a b}\right\} t_{m n o}^{e f c} \\
& \left(\Gamma_{(Q)}^{\prime}\right)_{j k}^{i a}=\left(\Gamma_{(Q)}^{\prime}\right)_{i a}^{j k}=-\frac{1}{24} \sum_{\text {efgmn }}\left\{\left(t^{[3]}\right)_{m n j k}^{\text {efga }}+\left(t^{\prime}\right)_{m n j k}^{\text {efga }}\right\} t_{m n i}^{\text {efg }}
\end{aligned}
$$

In the same way, the $\hat{T}_{4}^{[3]}$ and $T_{4}^{\prime}$ contributions to $\hat{S}_{3}$ may be computed at the same time. All other energy contributions and density matrix elements scale as $\mathscr{O}\left(n^{8}\right)$ or less.

For the $\operatorname{CCSDT}(\mathrm{Q}) / \mathrm{A}$ and $\operatorname{CCSDT}(\mathrm{Q}) / \mathrm{B}$ energies and gradients, the cost is increased somewhat over $\operatorname{CCSDT}(\mathrm{Q})$. In the case of $\operatorname{CCSDT}(\mathrm{Q}) / \mathrm{B}$, the introduction of $\hat{T}_{3}^{B}$ increases the number of $\mathscr{O}\left(n^{9}\right)$ steps to six for the energy, and to 16 for the gradient, since the density matrix contributions for $\hat{T}_{3}$ and $\hat{T}_{3}^{B}$ must be computed separately, and similarly for the contributions to $\hat{S}_{3}^{B}$. For $\operatorname{CCSDT}(\mathrm{Q}) / \mathrm{A}$, the cost is the same except that the $\hat{T}_{1}^{\dagger} \hat{T}_{2}^{\dagger}$ term could in theory be factorized such that it is computed at only $\mathscr{O}\left(n^{8}\right)$ cost, but to the authors' knowledge this has not been done in practice. The cost of a CCSDT(Q)/A,B energy or gradient is therefore intermediate between $\operatorname{CCSDT}(Q)$ and $\operatorname{CCSDT}(Q)_{\Lambda}$, where the latter requires the same number of $\mathscr{O}\left(n^{9}\right)$ steps as $\operatorname{CCSDT}(\mathrm{Q}) / \mathrm{B}$ but twice as many iterative $\mathscr{O}\left(n^{8}\right)$ equations. The analytic gradients of $\operatorname{CCSDT}(\mathrm{Q})_{\Lambda}$ will be studied in detail in a later publication.

In both $\operatorname{CCSDT}(\mathrm{Q})$ and $\operatorname{CCSDT}(\mathrm{Q}) / \mathrm{A}, \mathrm{B}$, the $\hat{T}_{4}^{[3],,, A, B}$ amplitudes need not be stored and may be computed on the fly for evaluation of the energy and contraction into the density matrices and $\hat{S}$ intermediates. However, unlike in $\operatorname{CCSD}(\mathrm{T})$, where no additional storage beyond that required for CCSD is needed, $\operatorname{CCSDT}(\mathrm{Q})$ does require the calculation of threeparticle intermediates,

$$
\begin{aligned}
\tilde{W}_{i j e}^{a b c} & =\frac{1}{2} P(a / b c) \sum_{f} v_{f e}^{b c} t_{i j}^{a f} \\
\tilde{W}_{i j k}^{a b m} & =-\frac{1}{2} P(i / j k) \sum_{n} v_{j k}^{n m} t_{i n}^{a b}+P(a / b) P(i j / k) \sum_{e} v_{e k}^{b m} t_{i j}^{a e}
\end{aligned}
$$


where the permutation operator $P$ antisymmetrizes the labels on either side of the slash. For $\operatorname{CCSDT}(\mathrm{Q})$ gradients, the similar intermediate three-particle density matrices $\tilde{\Gamma}_{b c d}^{a i j}$ and $\tilde{\Gamma}_{a b l}^{i j k}$ are also required. These intermediates must either be stored on disk or recalculated as needed. In our implementation we have found that storing them to disk tends to be the most efficient solution as recalculation is often more expensive and can, in extreme circumstances, even increase the formal scaling of the this term. However, as the $\tilde{W}_{i j e}^{a b c}$ intermediate in particular may be much larger than even the $\hat{T}_{3}$ amplitudes, the disk space requirements of CCSDT $(\mathrm{Q})$ calculations are indeed more strenuous than for CCSDT.

The iterative approximations all scale as $\mathscr{O}\left(n^{9}\right)$, with the rough order of cost given by CCSDTQ-3 > CC4 > CCSDTQ-1b > CCSDTQ-1a. The main feature that impacts the computational cost is the number of $\mathscr{O}\left(n^{9}\right)$ steps. For CCSDTQ-1a, the cluster amplitude equations require only four such steps per iteration, while all other methods require six steps due to the direct $\hat{T}_{4} \rightarrow \hat{T}_{3}$ coupling. The difference in the $\hat{\Lambda}$ equations is even larger: CCSDTQ-1a again requires four $\mathscr{O}\left(n^{9}\right)$ steps, but the other methods now require 12 steps per iteration due to the fact the the $\hat{T}_{4}$ amplitudes are required in addition to $\hat{\Lambda}_{4}$, and that these amplitudes are generally recomputed rather than stored due to their extremely large size. Additionally, the current implementations of the CCSDTQ-3 and CC4 $\hat{\Lambda}$ equations are sub-optimal in that they include two additional $\mathscr{O}\left(n^{9}\right)$ steps per iteration. The construction of the density matrices is not a major bottleneck for the iterative approximations because it occurs only once and not every iteration.

Many terms in the CCSDTQ-3 amplitude equations may be included by defining suitable two- and three-particle intermediates (including the seemingly expensive $\left(\hat{P}_{D} \hat{V} \hat{T}_{3} \hat{P}_{S}\right) \hat{T}_{3}$ term), but in particular the terms $t_{i j k l}^{a b c d} \leftarrow v_{e f}^{m n} t_{i j}^{a e} t_{k}^{f} t_{m n l}^{b c d}-v_{e f}^{m n} t_{i j}^{a e} t_{m}^{b} t_{n k l}^{f c d}$, while only scaling as $\mathscr{O}\left(n^{8}\right)$ involve contributions from $\hat{T}_{3}$ into an intermediate $\tilde{W}_{i j e}^{a b c}$. The large size of the inputs and outputs and relatively small size of the summation indices leads to low efficiency and a noticeably increased cost for CCSDTQ-3 compared to CCSDTQ-1b and CC4. The same is true of the contributions from a three-particle intermediate $\tilde{\Gamma}_{b c d}^{a i j}$ to the CCSDTQ-3 $\hat{\Lambda}_{3}$ 
equations. The iterative methods may also benefit from convergence acceleration via subiteration, $\underline{55,56}$ i.e. holding the $\hat{T}_{4}$ and/or $\hat{\Lambda}_{4}$ amplitudes constant while iteratively improving the other amplitudes. The contributions from the quadruples amplitudes may then be computed once per outer iteration and then added in at low cost during the inner iterations. In this work, sub-iterations is included in the cluster amplitude equations but not for the $\hat{\Lambda}$ amplitudes.

The accuracy of the present equations and implementation has been checked by comparing the computed gradients to finite differences of energies up to approximately $10^{-9}$ relative error in the molecular gradient where the finite difference method reaches the limit of its accuracy. The single-point energies have also been checked against the MRCC program of Kállay. $\stackrel{57}{\underline{n}}$ The construction of the one- and two-particle density matrices has also been checked by contracting them with the Hamiltonian to reproduce the various energies and/or energy corrections to numerical accuracy. A final check for the non-iterative approximations is contracting the $\hat{S}$ amplitudes with the corresponding $\hat{T}$ amplitudes, which should give the sum of all energy terms that include that $\hat{T}$ amplitude (perhaps with some redundancy). In

$\operatorname{CCSDT}(\mathrm{Q})$ for example, $\left\langle 0\left|\hat{S}_{2} \hat{T}_{2}\right| 0\right\rangle=\left\langle 0\left|\hat{T}_{2}^{\dagger} \hat{V} \hat{T}_{4}^{[3]}\right| 0\right\rangle+\left\langle 0\left|\hat{T}_{4}^{\prime}\left(\hat{V} \hat{T}_{2}^{2}\right)_{c}\right| 0\right\rangle$. The correctness of these expressions has also been checked.

\section{Results and Discussion}

The new implementations of approximate quadruples methods in CFOUR have been applied to two prototypical test systems: the isomerization of dimethylcarbene (DMC) to propene, and the simplest Criegee Intermediate (CI), $\stackrel{58}{\underline{4}} \mathrm{H}_{2}$ COO. In both cases, the optimized equilibrium and transition state structures and harmonic vibrational frequencies (via finite differences of gradients) have been determined at each level of theory, as well as with $\operatorname{CCSD}(\mathrm{T})$ and CCSDT for comparison. Absolute and relative (for the DMC-propene system) energies including harmonic vibrational zero-point energy were also calculated. In order to obtain 
an internally consistent benchmark and avoid complications with respect to basis set convergence, core correlation, relativistic effects, spin-orbit coupling, anharmonicity, etc., the computed values are compared against full CCSDTQ calculations instead of to experimental values. Core electrons are frozen in all calculations, and the double- $\zeta$ truncation of the ANO basis set of Almlöf and Taylor, $\underline{\underline{59}}$ often dubbed ANO0, is used. SCF, $\mathrm{CC} / \Lambda$, and geometry optimization thresholds were set to $10^{-10}, 10^{-9}$, and $10^{-8}$, respectively, with all other settings set to default. Harmonic vibrational frequencies for translational and rotational modes are below $0.2 \mathrm{~cm}^{-1}$ in all cases.

\subsection{Dimethylcarbene Isomerization}

The most stable conformation of singlet DMC is the $\mathrm{C}_{2}$ gauche conformation $(g$-DMC), $\underline{60}$ and the DMC and propene minima are connected by a single chiral transition state $(g$ TS) along the 1,2-hydrogen shift isomerization pathway. Table 1 lists the mean absolute errors for various categories of geometrical, energetic, and vibrational quantities compared to CCSDTQ. At each geometry, the error in the rotational constants rapidly decreases from 0.06-0.09\% down to essentially zero when full triple and then quadruple excitations are included. The same is true of the errors in bond lengths, although these are already fairly small at the $\operatorname{CCSD}(\mathrm{T})$ level $(\sim 30 \mathrm{fm})$. An exception is the non-bonded gauche $\mathrm{H}-\mathrm{H}$ distance in DMC $\left(\Delta r_{\mathrm{HH}}\right)$, which is quite sensitive to higher-order correlation effects. The bond and dihedral angle errors similarly tell a different story: the angle error at the CCSD(T) level increases dramatically from propene backwards along the isomerization pathway. For DMC, the mean absolute error is nearly $0.1^{\circ}$, and still $0.02^{\circ}$ at the transition state. For both $\mathrm{DMC}$ and $g$-TS, the angle error for the approximate quadruples methods is also higher than for propene by a factor of roughly five. Interestingly, CCSDT does not improve on $\operatorname{CCSD}(\mathrm{T})$ by this measure for propene or the transition state, but in contrast performs

fully as well as the approximate quadruples methods for DMC. The high angle error in the DMC structures, along with the spuriously large non-bonded $\mathrm{H}-\mathrm{H}$ distance seem to 
Table 1: Statistical errors in geometric, vibrational, and energetic properties for gauchedimethylcarbene $(g$-DMC), propene, and the hydrogen-shift transition state $(g$-TS). Errors in equilibrium rotational constants $(\triangle A B C)$ are listed as mean average percent errors (MAPE), while other values are listed as mean absolute errors (MAE). Bond length errors $(\Delta r)$ are in $\AA$, bond and dihedral angle errors $(\Delta L, \Delta \phi)$ are in degrees, harmonic frequency errors $(\Delta \omega)$ are in $\mathrm{cm}^{-1}$, and total $\left(\Delta E_{0}\right)$, relative $\left(\Delta T_{0}\right)$, and harmonic vibrational zero-point energy $(\triangle H V Z P E)$ errors are in $\mathrm{kcal} / \mathrm{mol}$. Total and relative energies include zero-point energy. The theoretical methods are listed in an abbreviated notation, e.g. Q-1a = CCSDTQ-1a.

(a) $g$-DMC

\begin{tabular}{|c||c|c|c|c|c|c|c|c|c|}
\hline & $(\mathrm{T})$ & $\mathrm{T}$ & $(\mathrm{Q})$ & $(\mathrm{Q}) / \mathrm{A}$ & $(\mathrm{Q}) / \mathrm{B}$ & $\mathrm{Q}-1 \mathrm{a}$ & $\mathrm{Q}-1 \mathrm{~b}$ & $\mathrm{Q}-3$ & $\mathrm{CC} 4$ \\
\hline \hline$\Delta A B C$ & $0.09 \%$ & $0.03 \%$ & $0.00 \%$ & $0.00 \%$ & $0.00 \%$ & $0.01 \%$ & $0.00 \%$ & $0.01 \%$ & $0.00 \%$ \\
\hline$\Delta r$ & 0.0003 & 0.0001 & 0.0000 & 0.0000 & 0.0000 & 0.0001 & 0.0000 & 0.0001 & 0.0000 \\
\hline$\Delta r_{\mathrm{HH}}$ & -0.0034 & -0.0003 & -0.0002 & -0.0001 & -0.0001 & 0.0000 & -0.0002 & -0.0001 & -0.0002 \\
\hline$\Delta \angle \Delta \phi$ & 0.087 & 0.003 & 0.006 & 0.002 & 0.004 & 0.014 & 0.006 & 0.002 & 0.006 \\
\hline$\Delta \omega$ & 2.11 & 1.06 & 0.11 & 0.05 & 0.04 & 0.97 & 0.05 & 0.47 & 0.07 \\
\hline$\Delta E_{0}$ & 1.337 & 0.507 & -0.017 & 0.019 & 0.006 & 0.394 & 0.009 & 0.198 & 0.005 \\
\hline$\Delta H V Z P E$ & 0.059 & 0.032 & -0.003 & 0.001 & 0.000 & 0.029 & 0.000 & 0.014 & -0.001 \\
\hline
\end{tabular}

(b) $g$-TS

\begin{tabular}{|c||l|l|l|l|l|l|l|l|l|}
\hline & $(\mathrm{T})$ & \multicolumn{1}{|c|}{$\mathrm{T}$} & \multicolumn{1}{|c}{$(\mathrm{Q})$} & $(\mathrm{Q}) / \mathrm{A}$ & $(\mathrm{Q}) / \mathrm{B}$ & $\mathrm{Q}-1 \mathrm{a}$ & $\mathrm{Q}-1 \mathrm{~b}$ & $\mathrm{Q}-3$ & $\mathrm{CC} 4$ \\
\hline \hline$\Delta A B C$ & $0.07 \%$ & $0.04 \%$ & $0.01 \%$ & $0.00 \%$ & $0.00 \%$ & $0.03 \%$ & $0.00 \%$ & $0.02 \%$ & $0.00 \%$ \\
\hline$\Delta r$ & 0.0004 & 0.0003 & 0.0001 & 0.0000 & 0.0000 & 0.0002 & 0.0000 & 0.0001 & 0.0000 \\
\hline$\Delta \angle \Delta \phi$ & 0.018 & 0.020 & 0.010 & 0.004 & 0.006 & 0.010 & 0.004 & 0.007 & 0.005 \\
\hline$\Delta \omega$ & 2.41 & 1.24 & 0.22 & 0.12 & 0.13 & 1.12 & 0.11 & 0.57 & 0.13 \\
\hline$\Delta E_{0}$ & 1.201 & 0.562 & -0.045 & -0.002 & -0.017 & 0.435 & -0.011 & 0.221 & -0.016 \\
\hline$\Delta T_{0}$ & -0.136 & 0.055 & -0.028 & -0.021 & -0.023 & 0.041 & -0.020 & 0.023 & -0.022 \\
\hline$\Delta H V Z P E$ & 0.061 & 0.036 & -0.006 & 0.001 & -0.002 & 0.032 & -0.001 & 0.016 & -0.002 \\
\hline
\end{tabular}

(c) Propene

\begin{tabular}{|c||l|l|l|l|l|l|l|l|l|}
\hline & $(\mathrm{T})$ & $\mathrm{T}$ & \multicolumn{1}{c}{$(\mathrm{Q})$} & $(\mathrm{Q}) / \mathrm{A}$ & $(\mathrm{Q}) / \mathrm{B}$ & $\mathrm{Q}-1 \mathrm{a}$ & $\mathrm{Q}-1 \mathrm{~b}$ & $\mathrm{Q}-3$ & $\mathrm{CC} 4$ \\
\hline \hline$\Delta A B C$ & $0.06 \%$ & $0.04 \%$ & $0.01 \%$ & $0.01 \%$ & $0.01 \%$ & $0.02 \%$ & $0.00 \%$ & $0.01 \%$ & $0.01 \%$ \\
\hline$\Delta r$ & 0.0002 & 0.0002 & 0.0000 & 0.0000 & 0.0000 & 0.0001 & 0.0000 & 0.0001 & 0.0000 \\
\hline$\Delta \angle \Delta \phi$ & 0.003 & 0.007 & 0.002 & 0.001 & 0.001 & 0.004 & 0.001 & 0.002 & 0.001 \\
\hline$\Delta \omega$ & 1.59 & 1.17 & 0.25 & 0.16 & 0.19 & 0.77 & 0.15 & 0.43 & 0.18 \\
\hline$\Delta E_{0}$ & 1.114 & 0.588 & -0.060 & -0.041 & -0.047 & 0.339 & -0.036 & 0.192 & -0.041 \\
\hline$\Delta T_{0}$ & -0.222 & 0.081 & -0.044 & -0.059 & -0.054 & -0.055 & -0.045 & -0.006 & -0.046 \\
\hline$\Delta H V Z P E$ & 0.048 & 0.035 & -0.008 & -0.005 & -0.006 & 0.023 & -0.005 & 0.013 & -0.005 \\
\hline
\end{tabular}


indicate that the major geometrical errors in DMC relate to non-bonding intermolecular interactions. As these interactions arise primarily form many-body effects, the fact that connected triple excitations (at least) are necessary to fully describe the structure is not surprising. An alternative hypothesis is that the biradical nature of DMC could account for the greater importance of higher-order correlation. However, a better description of biradical character would be expected to primarily affect the $\mathrm{C}-\mathrm{C}-\mathrm{C}$ angle. An inspection of the detailed data in the Supplemental Information shows that this is not the case, and that instead the largest angle errors are involved in the reorganization of the methyl hydrogens, especially the gauche hydrogen. This suggests that very high-accuracy structures with steric crowding (and potentially other strains such as ring strain) would benefit from the inclusion of quadruple excitations. Non-bonded intermolecular interactions are another area of possible applicability. Lane et al. calculated the quadruples contribution to the equilibrium geometry of the water dimer, $\underline{61}$ and while the effect on the intermolecular distance was small, there was a somewhat larger effect on the relative angles of the two water monomers (predominately the acceptor wag angle).

The harmonic vibrational frequencies for all three structures show a roughly order-ofmagnitude reduction in error for the approximate quadruples methods (with the exception of CCSDTQ-1a), while CCSDT roughly halves the error with respect to CCSD(T). Since $\operatorname{CCSD}(\mathrm{T})$ is in error approximately $2 \mathrm{~cm}^{-1}$, the inclusion of quadruple excitations is critical to achieving spectroscopic $\left(\mathrm{sub}-\mathrm{cm}^{-1}\right)$ accuracy. While inspection of the detailed results in the Supplemental Information shows that there are not any particular vibrational modes with excessively large error for DMC or propene, two modes of the transition state show errors in excess of $5 \mathrm{~cm}^{-1}$ at the $\operatorname{CCSD}(\mathrm{T})$ level, not surprisingly both involving movement of the migratory hydrogen. A highly-accurate description of these vibrational modes is essential to the description of kinetic tunneling, e.g. through semi-classical transition state theory. $\underline{62-64}$ Errors in the absolute energies are rather uninteresting, falling from just above 1 $\mathrm{kcal} / \mathrm{mol}$ down to approximately $0.2 \mathrm{~kJ} / \mathrm{mol}$ for the most accurate approximate quadruples 
methods (keep in mind that this is with respect to CCSDTQ, and errors compared to experiment will be larger). Outliers are CCSDTQ-1a and, more surprisingly, CCSDTQ-3, which quadruple or more the error of other approximate quadruples methods on average. Also to note is that the A and B variants of $\operatorname{CCSDT}(\mathrm{Q})$, while originally designed for open-shell ROHF calculations, also seem to slightly improve on "normal" CCSDT(Q) for these closedshell examples. When examining relative errors $\left(\Delta T_{0}\right)$ for the transition state and propene, $\operatorname{CCSD}(\mathrm{T})$ is seen to benefit greatly from error cancellation, bringing the average error down to $0.6-0.9 \mathrm{~kJ} / \mathrm{mol}$. While CCSDT similarly benefits from error cancellation, albeit to a lesser degree, only CCSDTQ-1a and CCSDTQ-3 gain any error cancellation benefit amongst the approximate quadruples methods. While this cancellation brings them in line with the other methods when considering relative energies, the comparatively poor behavior for absolute energies is rather troublesome and CCSDTQ-1a in particular should likely not be considered reliable.

\subsection{Criegee Intermediate}

Table 2: Statistical errors in geometric, vibrational, and energetic properties for CI. Errors in equilibrium rotational constants $(\triangle A B C)$ are listed as mean average percent errors (MAPE), while other values are listed as mean absolute errors (MAE). Bond length errors $(\Delta r)$ are in $\AA$, bond and dihedral angle errors $(\Delta L, \Delta \phi)$ are in degrees, harmonic frequency errors $(\Delta \omega)$ are in $\mathrm{cm}^{-1}$, and total $\left(\Delta E_{0}\right)$ and harmonic vibrational zero-point energy $(\triangle H V Z P E)$ errors are in $\mathrm{kcal} / \mathrm{mol}$. The total energy includes zero-point energy. The theoretical methods are listed in an abbreviated notation, e.g. Q-1a = CCSDTQ-1a.

\begin{tabular}{|c||l|l|l|l|l|l|l|l|l|}
\hline & $(\mathrm{T})$ & $\mathrm{T}$ & \multicolumn{1}{|c}{$(\mathrm{Q})$} & $(\mathrm{Q}) / \mathrm{A}$ & $(\mathrm{Q}) / \mathrm{B}$ & $\mathrm{Q}-1 \mathrm{a}$ & $\mathrm{Q}-1 \mathrm{~b}$ & $\mathrm{Q}-3$ & $\mathrm{CC} 4$ \\
\hline \hline$\Delta A B C$ & $0.34 \%$ & $0.27 \%$ & $0.72 \%$ & $0.26 \%$ & $0.35 \%$ & $0.33 \%$ & $0.04 \%$ & $0.11 \%$ & $0.04 \%$ \\
\hline$\Delta r$ & 0.0013 & 0.0018 & 0.0025 & 0.0011 & 0.0014 & 0.0013 & 0.0002 & 0.0004 & 0.0004 \\
\hline$\Delta \angle, \Delta \phi$ & 0.063 & 0.074 & 0.161 & 0.127 & 0.082 & 0.094 & 0.023 & 0.017 & 0.014 \\
\hline$\Delta \omega$ & 7.41 & 10.32 & 9.43 & 3.38 & 4.97 & 10.01 & 1.03 & 2.29 & 1.84 \\
\hline$\Delta E_{0}$ & 2.117 & 1.878 & -1.185 & 0.310 & -0.371 & 1.306 & -0.009 & 0.448 & -0.226 \\
\hline$\Delta H V Z P E$ & 0.060 & 0.089 & -0.102 & 0.021 & -0.049 & 0.076 & 0.006 & 0.024 & -0.011 \\
\hline
\end{tabular}

Mean absolute errors for CI are given in Table 2, Right off the bat, an inspection of the errors in the rotational constants, with errors approximately five times larger, show that 
CI is significantly more sensitive to higher-order correlation effects than the DMC-propene system. Increases in error of a similar magnitude are evident for bond lengths and angles, except that the angle error of DMC still outstrips that of CI at the CCSD(T) level. A major difference between DMC-propene and CI is that a number of higher-order methods fail to improve on CCSD(T) for these geometric quantities, with only CCSDTQ-1b and CC4 (and to some extent CCSDTQ-3) providing a reliable and significant decrease in error. Interestingly, $\operatorname{CCSDT}(\mathrm{Q})$ dramatically worsens all geometric errors by a factor of two; CCSDT(Q)/A and B improve somewhat but do not reach the accuracy of CCSDTQ-1b or CC4. In particular, the $\mathrm{O}-\mathrm{O}$ bond distance is vastly improved on going from $\operatorname{CCSD}(\mathrm{T})$ to the more accurate quadruples methods. On the other hand, the $\mathrm{C}-\mathrm{O}$ bond distance, which is well reproduced by $\operatorname{CCSD}(\mathrm{T})$, is problematic for $\operatorname{CCSDT}(\mathrm{Q})$ (but not A and B) and CCSDTQ-1a, and even for CCSDT. Among the angles, the $\mathrm{O}-\mathrm{O}-\mathrm{C}$ angle is clearly the most problematic, although several approximate quadruples methods as well as CCSDT worsen the $\mathrm{O}-\mathrm{C}-\mathrm{H}$ bond angles compared to $\operatorname{CCSD}(\mathrm{T})$. These results suggest that non-iterative approximations of quadruple excitations may not be able to reliably improve on $\operatorname{CCSD}(\mathrm{T})$ for describing the geometric parameters of moderately multi-configurational systems.

The harmonic frequencies show a similarly disappointing pattern, with CCSDT, CCSDT(Q), and CCSDTQ-1a showing a deterioration compared to the $\operatorname{CCSD}(\mathrm{T})$ values. Here, $\mathrm{CCSDT}(\mathrm{Q}) / \mathrm{A}$ and $\mathrm{B}$ are able to improve upon $\mathrm{CCSD}(\mathrm{T})$, with the A variant slightly in the lead. CCSDTQ$1 \mathrm{~b}$ and $\mathrm{CC} 4$, as before, again show a reliable reduction in error, although not to the sub- $\mathrm{cm}^{-1}$ level achieved for DMC-propene. A closer inspection of the data in the Supplemental Information shows that two frequencies, $\omega_{4}$ and $\omega_{6}$ are responsible for the lion's share of the error, especially at the $\mathrm{CCSD}(\mathrm{T})$ level. These frequencies correspond to $\mathrm{C}-\mathrm{O}$ and $\mathrm{O}-\mathrm{O}$ stretching vibrations. CCSDT and beyond tend to show an improvement in $\mathrm{O}-\mathrm{O}$ stretching, much as they show an improvement in the $\mathrm{O}-\mathrm{O}$ bond length, while $\mathrm{C}-\mathrm{O}$ stretching is worsened at the CCSDT level and to a slightly lesser extent at the CCSDT(Q) level. CCSDT, CCSDT(Q), and CCSDTQ-1a further exhibit severe errors (up to $30 \mathrm{~cm}^{-1}$ ) in the out-of-plane motions 
that are not present at the $\operatorname{CCSD}(\mathrm{T})$ level. It is quite difficult to ascribe these errors to a particular feature of the theory, for example using diagrammatic or perturbation arguments. Perhaps the simplest, albeit the least satisfying, explanation is a tendency of some of the methods, in particular CCSDT(Q), to "overshoot" the full CCSDTQ results. This can be seen explicitly in the absolute energies, where CCSDT(Q) overshoots CCSDTQ by more than a $\mathrm{kcal} / \mathrm{mol}$. On the other hand, CCSDT(Q)/A and /B under- and overestimate the CCSDTQ contribution by roughly the same amount, while CC4 also overshoots CCSDTQ. Thus, the extrapolation of simple energetics to geometric and vibrational parameters is qualitative at best. Perhaps the best example of the disconnect between energetics and geometry is seen for $\operatorname{CCSDT}(\mathrm{Q})$, which, as noted above, performs rather poorly for geometries and frequencies, but also halves the error in the absolute energy compared to CCSD(T). CCSDTQ-3 does make some improvement over CCSD(T) for CI, but is again not as accurate as CCSDTQ-1b.

\section{$4.3 \quad$ Timings}

While the results above show that certain iterative, and in some cases, non-iterative approximations to CCSDTQ can yield substantial accuracy gains compared to CCSD(T) and CCSDT, the computational cost of these methods is a critically important factor in determining when such methods may be used. Full CCSDTQ scales with the tenth power of system size $\left(\mathscr{O}\left(n^{10}\right)\right)$, and is applicable only to very small molecular systems, especially when analytic gradients or properties are desired. While the approximate methods described here reduce this scaling to $\mathscr{O}\left(n^{9}\right)$, they still represent a quite considerable cost increase over CCSDT and especially CCSD(T). Figure 1 shows timings for a single gradient evaluation, as a percentage of the CCSDTQ timing for all methods considered. CCSD(T) only requires $\sim 0.05 \%$ of the time compared to CCSDTQ, while CCSDT requires $\sim 0.4 \%$ of the CCSDTQ time. However, as CCSDT often fails to improve over $\operatorname{CCSD}(\mathrm{T})$, it is necessary to include approximate quadruple excitations if higher accuracy is required. CCSDT(Q) only increases the computational time over CCSDT modestly, keeping the relative timing under 


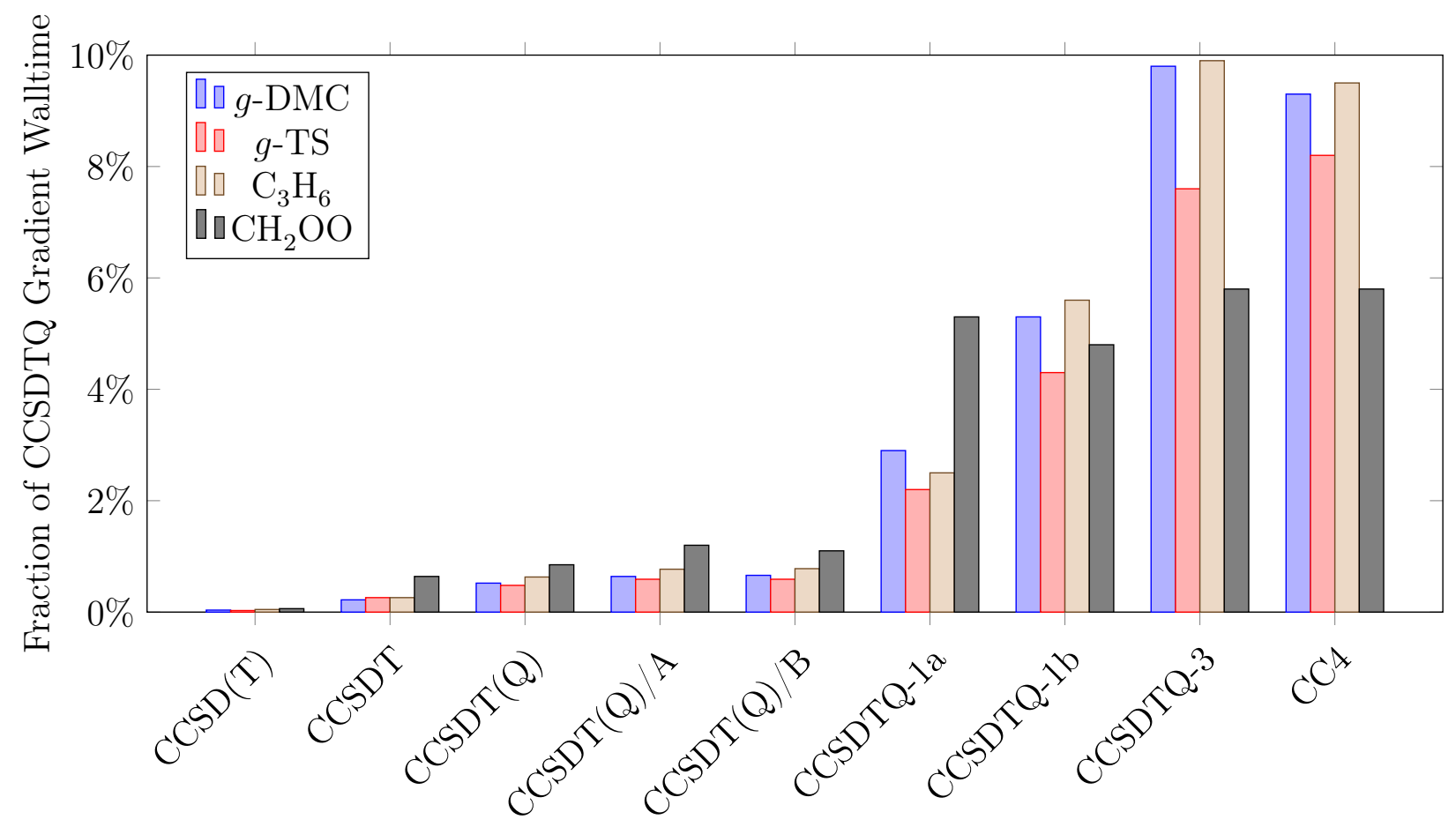

Figure 1: Timings (wall-time), relative to full CCSDTQ, for one gradient evaluation for all calculations presented in section 4.1 and section 4.2. All calculations utilized $2 \times \operatorname{Intel} \mathbb{R}$. Xeon E5-2695v4 $2.1 \mathrm{GHz}$ processors (36 cores total) with 256 GiB RAM. 
1\%. The A and $\mathrm{B}$ variants, while increasing the cost again slightly, likewise remain in the $1 \%$ range. When these methods can be considered reliable (strongly single-configurational systems with important many-body dynamic correlation), this modest increase in time over CCSDT for systems of the size studied here shows that they can be applied rather routinely. For CI, however, the non-iterative approximations are not reliable and an iterative approximations is mandatory. Of the iterative methods, CCSDTQ-1a is the cheapest, requiring 3-4\% of the CCSDTQ time. As shown above, however, this methods consistently fails to improve upon $\operatorname{CCSD}(\mathrm{T})$. CCSDTQ-1b, however, "only" increases the computational time to $4-5 \%$ of CCSDTQ, and performs perhaps the best among all of the approximate methods tested. CCSDTQ-3 and CC4 further increase the cost to between 6 and 10\% of CCSDTQ. CCSDTQ-3 is somewhat inconsistent in predicting both geometric and energetic quantities,

and due to its increased cost relative to CCSDTQ-1b, the former seems better justified as a standard iterative approximation. CC4 also performs consistently well, but again entails a cost increase over CCSDTQ-1b. Depending on whether an iterative or non-iterative approximation is appropriate for the system in question, a roughly one to two order-of-magnitude reduction in cost relative to full CCSDTQ can be expected, with very nearly the same level of accuracy.

\section{Conclusions}

The application of approximate quadruples methods to the equilibrium geometries and harmonic vibrational frequencies of the dimethylcarbene to propene isomerization pathway system shows a uniformly good comparison to the full CCSDTQ results, perhaps with the exception of the CCSDTQ-1a method. Since this method alone lacks a direct coupling from the quadruples amplitudes back to the triples, this seems the most likely explanation for this poor behavior. Even for DMC, which might be expected to exhibit mild multi-reference character due to the biradical carbon center, the performance of iterative and non-iterative 
approximate quadruples method alike remains very good. CCSD $(\mathrm{T})$ does indeed show an increase in error in this case, which can be traced almost exclusively to the interaction between the two gauche hydrogens which, presumably, has important many-body contributions. For these and, we predict, other "well-behaved" molecular systems, the CCSDT(Q) method, or one of the two variants developed to rigorously treat the case of a non-Hartree-Fock reference state, seem good choices that maximize the accuracy-to-computational cost ratio. In particular, based on the effect of non-bonded interactions in DMC, we expect that the inclusion of quadruples effects in the calculation of equilibrium geometries and (harmonic) vibrational frequencies to be important for dispersion-bound complexes and strained molecules.

The application to the simplest Criegee Intermediate is much more mixed, and clear evidence of multi-reference character is seen in e.g. the magnitude of the converged coupled cluster amplitudes. In this case, all methods exhibit a degradation of error with respect to CCSDTQ, but in particular, the CCSDT(Q) method (and to a lesser extent its A and B variants) as well as CCSDTQ-3 show a distinct worsening in accuracy relative to CCSD(T), CCSDT, and the other approximate quadruples methods. CCSDT(Q) performs especially poorly, and in fact worsens the agreement with full CCSDTQ compared to CCSD(T) in every regard except the total energy. Since the vast majority of previous studies so far have focused on the energy alone in evaluating $\operatorname{CCSDT}(\mathrm{Q})$ and other approximate quadruples methods, it seems important to consider derived properties in order to gain a full picture of the intrinsic accuracy of such methods. The results presented here suggest caution when applying CCSDT $(\mathrm{Q})$ to moderately multi-reference systems, although further benchmarking is necessary to fully delineate the realm of reasonable applicability. Luckily, the A and B variant show a distinct improvement over "plain" CCSDT(Q) even in the restricted Hartree-Fock case and serve as convenient substitutes. $\operatorname{CCSDT}(\mathrm{Q}) / \mathrm{A}$ can be implemented at essentially no additional cost compared to $\operatorname{CCSDT}(\mathrm{Q})$, although the current implementation in CFOUR is not optimal. Likewise CCSDTQ-1b is seen to perform well in all circumstances tested and could in some cases be justified as a higher-cost alternative. 
In sum, these results show that the application of post-CCSDT methods to the problem of equilibrium geometries, harmonic frequencies, and potentially to other properties as well is both feasible and potentially worthwhile when very high accuracy is necessary. Because of the computational cost of the CCSDTQ benchmark calculations, only molecules with three first-row atoms were investigated here. However, molecules with as many as six firstrow atoms should be accessible at the double- $\zeta$ level using the analytic gradient theory for approximate quadruples methods.

\section{Acknowledgement}

This work was supported by a generous start-up grant from SMU, and all calculations were performed on the ManeFrame II system at the SMU Center for Scientific Computation. I would like to specially thank Dr. John Stanton for his unrelenting encouragement, which was instrumental in bringing this work to completion.

\section{Supporting Information Available}

The following files are available free of charge. A supporting information file is available which contains individual errors for each geometrical and vibrational parameter as well as the CCSDTQ benchmark values.

\section{References}

(1) Martin, J. M. L. Annual Reports in Computational Chemistry; Elsevier, 2005; Vol. 1; pp 31-43.

(2) Feller, D.; Peterson, K. A.; Dixon, D. A. A Survey of Factors Contributing to Accurate Theoretical Predictions of Atomization Energies and Molecular Structures. J. Chem. Phys. 2008, 129, 204105. 
(3) Haunschild, R.; Klopper, W. New Accurate Reference Energies for the G2/97 Test Set. J. Chem. Phys. 2012, 136, 164102.

(4) Dixon, D. A.; Feller, D.; Peterson, K. A. In Annual Reports in Computational Chemistry; Wheeler, R. A., Ed.; Annual Reports in Computational Chemistry; Elsevier, 2012; Vol. 8; pp 1-28.

(5) Peterson, K. A.; Feller, D.; Dixon, D. A. Chemical Accuracy in Ab Initio Thermochemistry and Spectroscopy: Current Strategies and Future Challenges. Theor Chem Acc 2012, 131, 1079.

(6) Dixon, D. A.; Feller, D.; Peterson, K. A. Heats of Formation and Ionization Energies of NHx, X=0-3. J. Chem. Phys. 2001, 115, 2576-2581.

(7) Császár, A. G.; Leininger, M. L.; Szalay, V. The Standard Enthalpy of Formation of CH2. J. Chem. Phys. 2003, 118, 10631-10642.

(8) Feller, D.; Bross, D. H.; Ruscic, B. Enthalpy of Formation of N2H4 (Hydrazine) Revisited. J. Phys. Chem. A 2017, 121, 6187-6198.

(9) Karton, A.; Tarnopolsky, A.; Martin, J. M. L. Atomization Energies of the Carbon Clusters $\mathrm{C} n(\mathrm{n}=2-10)$ Revisited by Means of W4 Theory as Well as Density Functional, Gn, and CBS Methods. Mol. Phys. 2009, 10\%, 977-990,_eprint: https://doi.org/10.1080/00268970802708959.

(10) Ruden, T. A.; Helgaker, T.; Jørgensen, P.; Olsen, J. Coupled-Cluster ConnectedQuadruples Corrections to Atomization Energies. Chemical Physics Letters 2003, 371, $62-67$.

(11) Werner, H.-J.; Kállay, M.; Gauss, J. The Barrier Height of the F+H2 Reaction Revisited: Coupled-Cluster and Multireference Configuration-Interaction Benchmark Calculations. J. Chem. Phys. 2008, 128, 034305. 
(12) Hopkins, B. W.; Tschumper, G. S. Ab Initio Studies of $\Pi \cdots \pi$ Interactions: The Effects of Quadruple Excitations†. J. Phys. Chem. A 2004, 108, 2941-2948.

(13) Tajti, A.; Szalay, P. G.; Császár, A. G.; Kállay, M.; Gauss, J.; Valeev, E. F.; Flowers, B. A.; Vázquez, J.; Stanton, J. F. HEAT: High Accuracy Extrapolated Ab Initio Thermochemistry. J. Chem. Phys. 2004, 121, 11599-11613.

(14) Bomble, Y. J.; Vázquez, J.; Kállay, M.; Michauk, C.; Szalay, P. G.; Császár, A. G.; Gauss, J.; Stanton, J. F. High-Accuracy Extrapolated Ab Initio Thermochemistry. II. Minor Improvements to the Protocol and a Vital Simplification. J. Chem. Phys. 2006, $125,064108$.

(15) Harding, M. E.; Vázquez, J.; Ruscic, B.; Wilson, A. K.; Gauss, J.; Stanton, J. F. HighAccuracy Extrapolated Ab Initio Thermochemistry. III. Additional Improvements and Overview. J. Chem. Phys. 2008, 128, 114111.

(16) Thorpe, J. H.; Lopez, C. A.; Nguyen, T. L.; Baraban, J. H.; Bross, D. H.; Ruscic, B.; Stanton, J. F. High-Accuracy Extrapolated Ab Initio Thermochemistry. IV. A Modified Recipe for Computational Efficiency. J. Chem. Phys. 2019, 150, 224102.

(17) Martin, J. M. L.; de Oliveira, G. Towards Standard Methods for Benchmark Quality Ab Initio Thermochemistry-W1 and W2 Theory. J. Chem. Phys. 1999, 111, 1843-1856.

(18) Boese, A. D.; Oren, M.; Atasoylu, O.; Martin, J. M. L.; Kállay, M.; Gauss, J. W3 Theory: Robust Computational Thermochemistry in the kJ/Mol Accuracy Range. J. Chem. Phys. 2004, 120, 4129-4141.

(19) Karton, A.; Rabinovich, E.; Martin, J. M. L.; Ruscic, B. W4 Theory for Computational Thermochemistry: In Pursuit of Confident Sub-kJ/Mol Predictions. J. Chem. Phys. 2006, 125, 144108. 
(20) Klippenstein, S. J.; Harding, L. B.; Ruscic, B. Ab Initio Computations and Active Thermochemical Tables Hand in Hand: Heats of Formation of Core Combustion Species. $J$. Phys. Chem. A 2017, 121, 6580-6602.

(21) Raghavachari, K.; Trucks, G. W.; Pople, J. A.; Head-Gordon, M. A Fifth-Order Perturbation Comparison of Electron Correlation Theories. Chemical Physics Letters 1989, $157,479-483$.

(22) Noga, J.; Bartlett, R. J. The Full CCSDT Model for Molecular Electronic Structure. J. Chem. Phys. 1987, 86, 7041.

(23) Bomble, Y. J.; Stanton, J. F.; Kállay, M.; Gauss, J. Coupled-Cluster Methods Including Noniterative Corrections for Quadruple Excitations. Journal of Chemical Physics 2005, $123,4101$.

(24) Kucharski, S. A.; Bartlett, R. J. Recursive Intermediate Factorization and Complete Computational Linearization of the Coupled-Cluster Single, Double, Triple, and Quadruple Excitation Equations. Theor. Chem. Acc. Theory Comput. Model. Theor. Chim. Acta 1991, 80, 387-405.

(25) Oliphant, N.; Adamowicz, L. Coupled-cluster Method Truncated at Quadruples. J. Chem. Phys. 1991, 95, 6645-6651.

(26) Kucharski, S. A.; Bartlett, R. J. The Coupled-cluster Single, Double, Triple, and Quadruple Excitation Method. J. Chem. Phys. 1992, 97, 4282-4288.

(27) Morgan, W. J.; Matthews, D. A.; Ringholm, M.; Agarwal, J.; Gong, J. Z.; Ruud, K.; Allen, W. D.; Stanton, J. F.; Schaefer, H. F. Geometric Energy Derivatives at the Complete Basis Set Limit: Application to the Equilibrium Structure and Molecular Force Field of Formaldehyde. J. Chem. Theory Comput. 2018, 14, 1333-1350. 
(28) Puzzarini, C.; Heckert, M.; Gauss, J. The Accuracy of Rotational Constants Predicted by High-Level Quantum-Chemical Calculations. I. Molecules Containing First-Row Atoms. J. Chem. Phys. 2008, 128, 194108.

(29) Heckert, M.; Kállay, M.; Gauss, J. Molecular Equilibrium Geometries Based on Coupled-Cluster Calculations Including Quadruple Excitations. Mol. Phys. 2005, 103, 2109-2115,_eprint: https://doi.org/10.1080/00268970500083416.

(30) Heckert, M.; Kállay, M.; Tew, D. P.; Klopper, W.; Gauss, J. Basis-Set Extrapolation Techniques for the Accurate Calculation of Molecular Equilibrium Geometries Using Coupled-Cluster Theory. J. Chem. Phys. 2006, 125, 044108.

(31) Ruden, T. A.; Helgaker, T.; Jørgensen, P.; Olsen, J. Coupled-Cluster Connected Quadruples and Quintuples Corrections to the Harmonic Vibrational Frequencies and Equilibrium Bond Distances of HF, N2, F2, and CO. J. Chem. Phys. 2004, 121, 58745884 .

(32) Handy, N. C.; Schaefer, H. F. On the Evaluation of Analytic Energy Derivatives for Correlated Wave Functions. J. Chem. Phys. 1984, 81, 5031-5033.

(33) Kállay, M.; Gauss, J. Approximate Treatment of Higher Excitations in Coupled-Cluster Theory. II. Extension to General Single-Determinant Reference Functions and Improved Approaches for the Canonical Hartree-Fock Case. J. Chem. Phys. 2008, 129, 144101.

(34) Kállay, M.; Gauss, J. Approximate Treatment of Higher Excitations in Coupled-Cluster Theory. J. Chem. Phys. 2005, 123, 214105-214105-13.

(35) Gauss, J.; Stanton, J. F. Analytic Gradients for the Coupled-Cluster Singles, Doubles, and Triples (CCSDT) Model. J. Chem. Phys. 2002, 116, 1773.

(36) Kállay, M.; Gauss, J.; Szalay, P. G. Analytic First Derivatives for General CoupledCluster and Configuration Interaction Models. J. Chem. Phys. 2003, 119, 2991-3004. 
(37) Scuseria, G. E.; Schaefer, H. F. Analytic Evaluation of Energy Gradients for the Single, Double and Linearized Triple Excitation Coupled-Cluster CCSDT-1 Wavefunction: Theory and Applications. Chem. Phys. Lett. 1988, 146, 23-31.

(38) Gauss, J.; Stanton, J. F. Analytic First and Second Derivatives for the CCSDT-n $(\mathrm{N}=1-3)$ Models: A First Step towards the Efficient Calculation of CCSDT Properties. Phys. Chem. Chem. Phys. 2000, 2, 2047-2060.

(39) Scuseria, G. E. Analytic Evaluation of Energy Gradients for the Singles and Doubles Coupled Cluster Method Including Perturbative Triple Excitations: Theory and Applications to FOOF and Cr2. J. Chem. Phys. 1991, 94, 442.

(40) Watts, J. D.; Gauss, J.; Bartlett, R. J. Open-Shell Analytical Energy Gradients for Triple Excitation Many-Body, Coupled-Cluster Methods: MBPT(4), CCSD $+\mathrm{T}(\mathrm{CCSD}), \mathrm{CCSD}(\mathrm{T})$, and QCISD(T). Chemical Physics Letters 1992, 200, 1-7.

(41) Scheiner, A. C.; Scuseria, G. E.; Rice, J. E.; Lee, T. J.; Schaefer, H. F. Analytic Evaluation of Energy Gradients for the Single and Double Excitation Coupled Cluster (CCSD) Wave Function: Theory and Application. J. Chem. Phys. 1987, 87, 5361.

(42) Rendell, A. P.; Lee, T. J. An Efficient Formulation and Implementation of the Analytic Energy Gradient Method to the Single and Double Excitation Coupled-Cluster Wave Function: Application to Cl2O2. J. Chem. Phys. 1991, 94, 6219.

(43) Gauss, J.; Stanton, J. F.; Bartlett, R. J. Coupled-cluster Open-shell Analytic Gradients: Implementation of the Direct Product Decomposition Approach in Energy Gradient Calculations. The Journal of Chemical Physics 1991, 95, 2623-2638.

(44) Čižzek, J. On the Correlation Problem in Atomic and Molecular Systems. Calculation of Wavefunction Components in Ursell-Type Expansion Using Quantum-Field Theoretical Methods. J. Chem. Phys. 1966, 45, 4256-4266. 
(45) Shavitt, I.; Bartlett, R. J. Many-Body Methods in Chemistry and Physics: MBPT and Coupled-Cluster Theory, 1st ed.; Cambridge University Press: Cambridge ; New York, 2009 .

(46) Helgaker, T.; Jorgensen, P.; Olsen, J. Molecular Electronic-Structure Theory, 1st ed.; Wiley: Chichester ; New York, 2013.

(47) Rice, J. E.; Amos, R. D.; Handy, N. C.; Lee, T. J.; Schaefer, H. F. The Analytic Configuration Interaction Gradient Method: Application to the Cyclic and Open Isomers of the S3 Molecule. J. Chem. Phys. 1986, 85, 963-968.

(48) Bartlett, R. J.; Watts, J. D.; Kucharski, S. A.; Noga, J. Non-Iterative Fifth-Order Triple and Quadruple Excitation Energy Corrections in Correlated Methods. Chemical Physics Letters 1990, 165, 513-522.

(49) Stanton, J. F. Why CCSD(T) Works: A Different Perspective. Chem. Phys. Lett. 1997, 281, 130 .

(50) Matthews, D. A.; Stanton, J. F. Non-Orthogonal Spin-Adaptation of Coupled Cluster Methods: A New Implementation of Methods Including Quadruple Excitations. J. Chem. Phys. 2015, 142, 064108.

(51) Stanton, J. F.; Gauss, J.; Cheng, L.; Harding, M. E.; Matthews, D. A.; Szalay, P. G.; Auer, A. A.; Bartlett, R. J.; Benedikt, U.; Berger, C.; Bernholdt, D. E.; Bomble, Y. J.; Christiansen, O.; Engel, F.; Faber, R.; Heckert, M.; Heun, O.; Hilgenberg, M.; Huber, C.; Jagau, T.-C.; Jonsson, D.; Jusełius, J.; Kirsch, T.; Klein, K.; Lauderdale, W. J.; Lipparini, F.; Metzroth, T.; Mück, L. A.; O’Neill, D. P.; Price, D. R.; Prochnow, E.; Puzzarini, C.; Ruud, K.; Schiffmann, F.; Schwalbach, W.; Simmons, C.; Stopkowicz, S.; Tajti, A.; Vázquez, J.; Wang, F.; Watts, J. D. CFOUR, Coupled-Cluster Techniques for Computational Chemistry, a Quantum-Chemical Program Package with the Integral 
Packages MOLECULE (J. Almlöf and P. R. Taylor), PROPS (P. R. Taylor), ABACUS (T. Helgaker, H. J. Jensen, P. Jørgensen and J. Olsen), and ECP Routines by A. V. Mitin and C. van Wüllen; www.cfour.de.

(52) Matthews, D. A.; Stanton, J. F. In Mathematical Physics in Theoretical Chemistry; Blinder, S. M., House, J. E., Eds.; Developments in Physical \& Theoretical Chemistry; Elsevier, 2019; pp 327-375.

(53) Matthews, D. High-Performance Tensor Contraction without Transposition. SIAM J. Sci. Comput. 2018, 40, C1-C24.

(54) Matthews, D. A. On Extending and Optimising the Direct Product Decomposition. Mol. Phys. 2019, 117, 1325-1333.

(55) Matthews, D. A.; Stanton, J. F. Accelerating the Convergence of Higher-Order Coupled Cluster Methods. J. Chem. Phys. 2015, 143, 204103.

(56) Matthews, D. Accelerating the Convergence of Higher-Order Coupled Cluster Methods II: Coupled Cluster $\Lambda$ Equations and Dynamic Damping. Mol. Phys. 2020,

(57) Kállay, M.; Nagy, P. R.; Mester, D.; Rolik, Z.; Samu, G.; Csontos, J.; Csóka, J.; Szabó, P. B.; Gyevi-Nagy, L.; Hégely, B.; Ladjánszki, I.; Szegedy, L.; Ladóczki, B.; Petrov, K.; Farkas, M.; Mezei, P. D.; Ganyecz, Á. The MRCC Program System: Accurate Quantum Chemistry from Water to Proteins. J. Chem. Phys. 2020, ESS2020, 074107.

(58) Criegee, R.; Wenner, G. Die Ozonisierung Des 9,10-Oktalins. Justus Liebigs Annalen der Chemie 1949, 564, 9-15.

(59) Almlöf, J.; Taylor, P. R. In Advances in Quantum Chemistry; Löwdin, P.-O., Sabin, J. R., Zerner, M. C., Eds.; Academic Press, 1991; Vol. 22; pp 301-373. 
(60) Richards, C. A.; Kim, S.-J.; Yamaguchi, Y.; Schaefer, H. F. Dimethylcarbene: A Singlet Ground State? J. Am. Chem. Soc. 1995, 117, 10104-10107.

(61) Lane, J. R. CCSDTQ Optimized Geometry of Water Dimer. J. Chem. Theory Comput. 2013, 9, 316-323.

(62) Miller, W. H. Semiclassical Limit of Quantum Mechanical Transition State Theory for Nonseparable Systems. J. Chem. Phys. 1975, 62, 1899-1906.

(63) Miller, W. H. Quantum and Semiclassical Theory of Chemical Reaction Rates. Faraday Discuss. 1998, 110, 1-21.

(64) Nguyen, T. L.; Stanton, J. F.; Barker, J. R. A Practical Implementation of SemiClassical Transition State Theory for Polyatomics. Chemical Physics Letters 2010, 499, 9-15. 